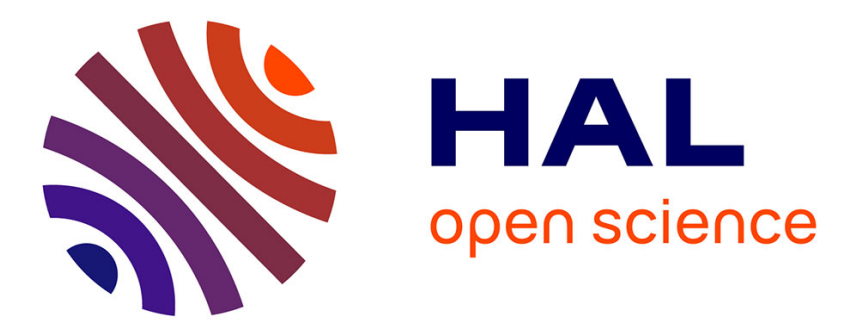

\title{
Multiparametric characterization of neuronal subpopulations in the ventrolateral preoptic nucleus
}

Romain Dubourget, Aude Sangare, Hélène Geoffroy, Thierry Gallopin, Armelle Rancillac

\section{- To cite this version:}

Romain Dubourget, Aude Sangare, Hélène Geoffroy, Thierry Gallopin, Armelle Rancillac. Multiparametric characterization of neuronal subpopulations in the ventrolateral preoptic nucleus. Brain Structure and Function, 2017, 222 (3), pp.1153-1167. 10.1007/s00429-016-1265-2 . inserm-02126237

\section{HAL Id: inserm-02126237 https://www.hal.inserm.fr/inserm-02126237}

Submitted on 11 May 2019

HAL is a multi-disciplinary open access archive for the deposit and dissemination of scientific research documents, whether they are published or not. The documents may come from teaching and research institutions in France or abroad, or from public or private research centers.
L'archive ouverte pluridisciplinaire HAL, est destinée au dépôt et à la diffusion de documents scientifiques de niveau recherche, publiés ou non, émanant des établissements d'enseignement et de recherche français ou étrangers, des laboratoires publics ou privés.

\section{(ㅇ)(1) $\$$}

Distributed under a Creative Commons Attribution - NonCommercial - NoDerivatives| 4.0 


\title{
Multiparametric characterization of neuronal subpopulations in the ventrolateral preoptic nucleus
}

\author{
Romain Dubourget $^{1,2}$, Aude Sangaré ${ }^{1,2}$, Hélène Geoffroy ${ }^{1,2}$, Thierry Gallopin ${ }^{1,2}$ \& Armelle \\ Rancillac $^{1,2 * \dagger}$
}

${ }^{1}$ Brain Plasticity Unit, ESPCI-ParisTech, PSL Research University, Paris, F-75005, France.

${ }^{2}$ CNRS, UMR 8249, Paris, F-75005, France.

$†$ Present address: Armelle Rancillac, CIRB, Collège de France, CNRS UMR 7241 /Inserm U1050

Abbreviated title: Unsupervised clustering of VLPO neurons.

Keywords: Sleep-promoting neuron, serotonin, noradrenaline, VLPO, Ward's clustering, low-threshold calcium spike (LTS).

* Correspondence should be addressed to:

Dr. Armelle Rancillac

Brain Plasticity Unit

CNRS UMR 8249, ESPCI ParisTech

10 rue Vauquelin, 75005 Paris, France.

Tel.: +33(0)1 40795183

Fax: +33(0)140794757

E-mail: armelle.rancillac@college-de-france.fr 


\begin{abstract}
The characterization of neuronal properties is a necessary first step toward understanding how the ventrolateral preoptic nucleus (VLPO) neuronal network regulates slow-wave sleep (SWS). Indeed, the electrophysiological heterogeneity of VLPO neurons suggests the existence of subtypes that could differently contribute in SWS induction and maintenance. The aim of the present study was to define cell classes in the VLPO using an unsupervised clustering classification method. Electrophysiological features extracted from 289 neurons recorded in whole-cell patch-clamp allowed the identification of three main classes of VLPO neurons subdivided into 5 distinct subpopulations (cluster 1, 2a, 2b, 3a and 3b). The high occurrence of a low-threshold calcium spike (LTS) was one of the most distinctive features of cluster 1 and 3. Since sleep-promoting neurons are generally identified by their ability to generate an LTS and by their inhibitory response to noradrenaline (NA), 189 neurons from our dataset were also tested for this neurotransmitter. Neurons from cluster 3 were the most frequently inhibited by NA. Biocytin labeling and Neurolucida reconstructions of 112 neurons furthermore revealed a small dendritic arbor of cluster $3 b$ neurons compared, in particular, to cluster $2 b$ neurons. Altogether, we performed an exhaustive characterization of VLPO neuronal subtypes that is a crucial step toward a better understanding of the neuronal network within the VLPO and thereby sleep physiology.
\end{abstract}




\section{Introduction}

Compared to the large number of cell groups involved in arousal, relatively few populations of neurons have been shown to turn off the arousal system. In the anterior hypothalamus, the ventrolateral preoptic nucleus (VLPO) is considered as the main brain region responsible for the inhibition of wake-promoting neurons during slow-wave sleep (SWS) (Von Economo 1930; Sherin et al. 1996; Saper et al. 2010). Indeed, retrograde transport experiments identified a set of GABAergic neurons in the VLPO, containing the peptide galanin, which innervate all of the major components of the ascending arousal systems (Sherin et al. 1998; Saper et al. 2005).

Two main classes of neurons have already been characterized, ex vivo in the VLPO of rats. The largest neuronal type displays triangular-shape, expresses galanin and is endowed with the ability to generate a low-threshold calcium spike (LTS). Moreover, these neurons are inhibited by several arousal neurotransmitters such as noradrenaline (NA) (Gallopin et al. 2000; Matsuo et al. 2003; Moore et al. 2012; Liu et al. 2013; McCarren et al. 2014; Varin et al. 2015; Scharbarg et al. 2016), suggesting that they are maintained silent during waking. Thus, they are named NA (-) neurons and considered as putative sleep-promoting neurons. Two intermingled subtypes of NA (-) neurons were later demonstrated to differ in their firing responses to serotonin (5-HT). Indeed, in contrast to other neurotransmitters that promote wakefulness, 5HT was shown to inhibit (44\%; Type-1) or to excite (56\%; Type-2) NA (-) neurons, revealing two potential different neuronal subtypes of putative sleep-promoting neurons (Gallopin et al. 2005; Fort et al. 2009).

Albeit far less studied, VLPO neurons excited by NA (i.e. NA (+) neurons) have only been sparsely described at the present time. In rats, they display a fusiform/bipolar shape and were described as devoid of LTS (Gallopin et al. 2000; Matsuo et al. 2003; Liu et al. 2010). These 
neurons are supposed to correspond to local neurons and are considered as non associated with sleep promotion.

To describe the neuronal diversity within the VLPO, here we first performed an exhaustive electrophysiological characterization of the neurons. Unsupervised clustering analysis based on these electrophysiological features revealed 3 different main classes and 5 subclasses of VLPO neurons. We furthermore determined their pharmacological responses to bath application of NA and 5-HT and performed Neurolucida reconstructions of the biocytin-filled VLPO neurons to reveal distinctive physiological and morphological properties among these neuronal subtypes. Altogether, our results establish the existence of a complex neuronal diversity within the VLPO that represents an important step toward understanding sleep regulation. 


\section{Materials and Methods}

Animals.99 Male-only C57BL/6J mice (14-21 days old; Charles River, France) were used for all experiments. Mice arrived in the laboratory at least 1 week before experiments to acclimate to their new environment, and were housed in a temperature-controlled $\left(20-22^{\circ} \mathrm{C}\right)$ room under a 12-hour light-dark cycle (lights on at 09:00 a.m.) with ad libitum access to food and water. All animal procedures were conducted in strict compliance with our institutional protocols and were approved by the European Community Council Directive of 22 September 2010 (010/63/UE) and the local ethics committee (Comité d'éthique en matière d'expérimentation animale number 59, C2EA-59, 'Paris Centre et Sud'). The number of animals in our study was accordingly kept to the necessary minimum.

Preparation of acute hypothalamic slices. Animals were decapitated at the beginning of the light phase, between 09:00 and 10:00 a.m. Brains were quickly extracted without removing the meninges and submerged in cold slicing artificial cerebrospinal fluid (aCSF, $4^{\circ} \mathrm{C}$ ) containing (in mM): $130 \mathrm{NaCl} ; 5 \mathrm{KCl} ; 2.4 \mathrm{CaCl}_{2} ; 20 \mathrm{NaHCO}_{3} ; 1.25 \mathrm{KH}_{2} \mathrm{PO}_{4} ; 1.3 \mathrm{MgSO}_{4} ; 10$ D-glucose; 15 sucrose; and 1 kynurenic acid $(\mathrm{pH}=7.35)$. Brains were constantly oxygenated with $95 \% \mathrm{O}_{2}-$ $5 \% \mathrm{CO}_{2}$. Coronal brain slices ( $300 \mu \mathrm{m}$ thick) containing the VLPO were cut with a vibratome (VT2000S; Leica) and transferred to a constantly oxygenated $\left(95 \% \mathrm{O}_{2}-5 \% \mathrm{CO}_{2}\right)$ holding chamber containing aCSF. Subsequently, individual slices were placed in a submerged recording chamber maintained at $32^{\circ} \mathrm{C}$ and perfused with oxygenated kynurenate-free aCSF, and placed under an upright microscope (Zeiss) equipped with a CCD camera (Hamamatsu) for observation.

Drugs. Noradrenaline (NA, $50 \mu \mathrm{M}$; Sigma) and serotonin (5-HT, $100 \mu \mathrm{M}$; Sigma) were used in this study. 
Whole-cell patch-clamp recordings. Slices were maintained immersed and continuously surperfused at 1-2 mL/min with oxygenated kynurinic acid-free aCSF. Electrophysiological experiments were performed with a MultiClamp700B (Axon Instruments) amplifier connected to an acquisition board (Digidata 1440; Axon Instruments) attached to a computer running the pCLAMP software (Axon Instruments). The targeted neurons were located in the VLPO.

Recordings in the whole-cell patch-clamp configuration were performed with patch-clamp pipettes (3-6 $\mathrm{M} \Omega$ ) filled with an internal solution containing (in $\mathrm{mM}$ ): $144 \mathrm{~K}$-gluconate; $1 \mathrm{MgCl}_{2} ; 0.5$ EGTA; 10 HEPES (pH 7.2); and 285-295 mOsm for 126 recorded neurons or with an internal solution containing (in $\mathrm{mM}$ ): $144 \mathrm{~K}$-gluconate; $3 \mathrm{MgCl}_{2} ; 0.2$ EGTA; 10 HEPESfor 163 recorded neurons. Electrophysiological features of these two populations were compared among all neurons and within each cluster, and no statistical differences were found. For morphological analysis, $2 \mathrm{mg} / \mathrm{mL}$ biocytin (Sigma Aldrich) was added to these internal solutions. The liquid junction potential of the patch pipette and the perfused extracellular solution were $11 \mathrm{mV}$ and were not applied to the data. The pipette was slowly brought to the selected neuron for recording in whole-cell configuration using infrared videomicroscopy guidance and clamped at $-60 \mathrm{mV}$. The intrinsic neuronal membrane properties were assessed in current-clamp by applying current steps $(800 \mathrm{~ms})$ from -100 pA until firing saturation, in $10 \mathrm{pA}$ increments.

For each neuron, 39 electrophysiological parameters were measured according to the Petilla terminology (Ascoli et al. 2008), as fully described in (Karagiannis et al. 2009). Briefly, (1) the resting membrane potential was measured immediately after passing to whole-cell configuration. (2) Input resistance $(\mathrm{Rm})$ and (3) the membrane time constant $(\tau \mathrm{m})$ were determined at the beginning of the voltage response of a hyperpolarization current step (10 pA, $800 \mathrm{~ms}$ ). The time constant was determined by fitting this voltage response to a single 
exponential. (4) The membrane capacitance $(\mathrm{Cm})$ was calculated according to the equation $\mathrm{Cm}=\tau \mathrm{m} / \mathrm{Rm}$. Under our conditions, injection of hyperpolarizing current pulses often induced a hyperpolarization-activated cationic current (Ih) that followed the initial hyperpolarization peak, known as a sag. (5) Ghyp was measured as the slope of the linear portion of an I-V plot, measured at the beginning $(0-0.1 \mathrm{~s} ;-100$ to $0 \mathrm{pA})$. (6) $\mathrm{G}_{\text {sag }}$ was measured as the slope of the linear portion of a current-voltage (I-V) plot, measured at the end of the hyperpolarizing current pulses (0.7-0.8 s; -100 to $0 \mathrm{pA})$. (7) $\Delta \mathrm{G}_{\text {Sag }}$ corresponds to $\left(\mathrm{G}_{\mathrm{sag}}-\mathrm{G}_{\mathrm{hyp}}\right) / \mathrm{G}_{\text {sag. }}$. (8) Action potential threshold corresponded to the voltage threshold of the first action potential elicited by a current ramp. (9) The rheobase was the minimum current that elicited an action potential. (10) The first spike latency was computed as the time needed to elicit a spike after the onset of a current pulse corresponding to the rheobase. Neurons have been described to exhibit a wide range of firing behaviors around threshold, some exhibiting bursting, adapting, regular or irregular trains of action potentials. In order to describe this variety of behaviors with quantitative parameters, the interspaced intervals measured in response to the minimal current injection eliciting more than three action potentials were plotted and fitted to a linear curve. (11) Adaptation ( $m_{\text {threshold }}$ ) was computed as the slope of the linear fit and (12) the minimal steady state frequency ( $\left.F_{\text {threshold }}\right)$. (13) The spontaneous discharge was measured in the cellattached configuration, just before the patch break down. When depolarized by current injection, VLPO neurons displayed several action potential discharged patterns. Initial burst of action potentials followed by a shorter 'interspike interval' than the steady-state trains of spikes was referred as (14) burst firing neurons. We also observed (15) non-adapting non-fast spiking firing pattern, (16) adapting firing, (17) late spiking firing and (18) irregular firing. (19 and 23) Amplitudes of the first (A1) and second (A2) action potential (AP) were measured from the threshold to the positive peak on the first step, where at least 3 APs were induced. (20 and 24) AP durations were measured at half-amplitude (D1 and D2). (21) The amplitude (AHP $\max$ 
amplitude) and (22) duration of the maximal after-hyperpolarization ( $\left.\mathrm{AHP}_{\max }\right)$ were measured for the first AP. (25) Amplitude variation (Var A) and (26) duration variation (Var D) were computed as (A1-A2)/A1*100 and (D2-D1)/D1*100 respectively. At higher stimulation intensities, the maximal firing rate was defined as the last trace before prominent reduction of the action potential amplitude, indicative of a saturated discharge. To take into account the biphasic spike frequency adaptation (early and late), instantaneous firing frequency was fitted to a single exponential with a sloping baseline, according to the equation $\mathrm{F}_{\mathrm{sat}}=\mathrm{A}_{\mathrm{sat}} \times \mathrm{E}^{-\mathrm{t} / \mathrm{sat}}+\mathrm{t} \times \mathrm{m}_{\text {sat }}+\mathrm{F}_{\mathrm{max}}$, where (27) $\mathrm{A}_{\text {sat }}$ corresponds to the amplitude of early frequency adaptation, (28) $\tau_{\text {sat }}$ to the time constant of early adaptation, (29) $F_{\max }$ the maximal steady-state frequency and (30) $\mathrm{m}_{\text {sat }}$ to the slope of late adaptation. LTS VLPO neurons are believed to be the putative sleep-promoting. In addition to the Petilla terminology, current pulses of increased amplitude were applied on neurons hold at $<-80 \mathrm{mV}$ to determine the presence or absence of an (31) LTS. (32) LTS amplitude and (33) duration were also measured on the first step that elicited an LTS. (34) The number of AP elicited by the LTS (AP/burst) was counted and (35) the outward rectification in the positive direction (Slop) was measured. (36) Occurrence of post-inhibitory rebound, (37) its amplitude, (38) duration and (39) the number of AP elicited by a post-inhibitory rebound following hyperpolarizing current of 100 pA from $-60 \mathrm{mV}$ were measured.

Pharmacological responses to NA and 5-HT applications were recorded in current-clamp mode. Data were rejected if the access resistance increased over $25 \mathrm{MOhm}$ during a recording or changed by more than $25 \%$ during the recording.

Ward's Clustering and Silhouette. Unsupervised clustering was performed essentially as described previously (Karagiannis et al. 2009; Perrenoud et al. 2012). Briefly, 39 electrophysiological parameters were standardized by centering and reducing all of the values 
of the 289 neurons using Statistica 6 software (Statsoft). After the selection of significant principal components (principal component analysis with absolute loading values of 0.7 ), we conducted a cluster analysis using unsupervised clustering performed by Ward's method (Ward 1963) that does not require to set a number of classes to be characterized. The clusters generated by Ward's method were then corrected using the K-means algorithm by using Matlab software (MathWorks). To quantitatively assess the quality of a clustering, silhouette analysis of the Kmeans clustering was performed. A positive silhouette value indicates that on average, the neuron is closer to the neurons of its own cluster than from the neurons belonging to other clusters in the parameter space. On the opposite, a negative value indicates a potential misclassification. The 18 electrophysiological parameters used for clustering analysis were $\mathrm{R}_{\mathrm{m}}$ (2), $\tau_{\mathrm{m}}(3), \mathrm{C}_{\mathrm{m}}(4), \Delta \mathrm{G}_{\text {Sag }}(7)$, first spike latency (10), $\mathrm{m}_{\text {threshold }}(11), \mathrm{F}_{\text {threshold }}$ (12), bursting firing

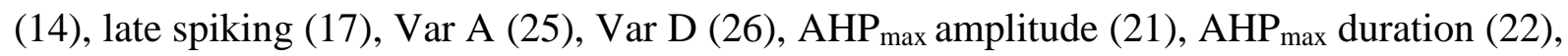
$\mathrm{m}_{\text {sat }}$ (30), LTS occurrence (31), rebound amplitude (37), rebound duration (38), and number of AP elicited by rebound (39).

Morphological analysis. In order to describe variations of morphology between recorded neurons, a total of 40 parameters related to features of the soma, dendrites and axon were extracted. Morphologies of somata were reconstructed from infrared pictures taken prior to whole cell recording, while in the cell attached configuration with a CCD camera controlled by the Image-Pro 7 software (Media Cybernetics Inc.). Morphological features were measured from these images after calibration with a standard $24 \mu \mathrm{m}$ grid. (40) The cell body area, (41) perimeter, (42) form factor, and (43) maximal, (44) minimal diameter passing through the centroid were computed. The degree of flatness of a contour shape, taken as the ratio of its minimum diameter to its maximum diameter, was indicated as (45) the aspect ratio. (46) The somatic solidity, the ratio of the somata area as a whole over the convex area, where values 
closer to 1 represent more solid (i.e. smooth, uniform) somata was calculated for each soma. (47) Convexity $=($ Convex Perimeter $) /($ Perimeter $)$. This parameter is indicative of the somatic morphological profile. The measurement of how closely this shape approached that of a circle was assessed throughout (48) the roundness. Finally, (49) somatic compactness $=[\sqrt{ }(4 / \pi) \times$ area $] /($ feret max $)$; numerical values of somatic compactness closer to 1 represent a more compact soma.

The dendritic properties of recorded neurons were extracted by using Neurolucida explorer (MBF Bioscience) and Excel (Microsoft). In order to be able to describe the differences of dendritic arborizations, we systematically quantified the following parameters: (50) Number of primary dendrites, (51) total dendritic length, (52) total dendritic surface, (53) total dendritic volume, (54) ratio of the total dendritic length on the total dendritic surface, (55) highest dendritic order segment, (56) average tortuosity of dendritic segments (defined as the ratio of the length of the segment over the straight line path between its extremities) and (57) number of dendritic nodes. We performed a Sholl analysis (Sholl 1953) to describe differences between neuronal arborization. (58) The length of the dendritic arborization that could be enclosed within a $100 \mu \mathrm{m}$ radius circle around the cell body, (59) between $100 \mu \mathrm{m}$ and $200 \mu \mathrm{m}$ radii, (60) between $200 \mu \mathrm{m}$ and $300 \mu \mathrm{m}$ radii, and (61) outside a $300 \mu \mathrm{m}$ radius was systematically extracted. Finally, (62-67) the number of dendritic spines in each segment order was extracted and (68) mean spine density was calculated.

Similarly, axonal properties were also extracted when the total axonal length of the reconstructed neurons exceeded $1 \mathrm{~mm}$. (69) the percentage of axonal reconstruction per cluster was calculated. (70) Axonal length, (71) total axonal surface, (72) total axonal volume, (73) ratio of the total axonal length on the total axonal surface, (74) average tortuosity of axonal segments and (75) number of axonal nodes. We performed a Sholl analysis of digitized axonal morphology. Axonal length that could be enclosed within a $100 \mu \mathrm{m}$ radius circle around the 
cell body, (76) between $100 \mu \mathrm{m}$ and $200 \mu \mathrm{m}$ radii, (77) between $200 \mu \mathrm{m}$ and $300 \mu \mathrm{m}$ radii, (78) between $300 \mu \mathrm{m}$ and $400 \mu \mathrm{m}$ radii, and (79) outside a $400 \mu \mathrm{m}$ radius was systematically extracted.

Spontaneous excitatory and inhibitory currents. Spontaneous currents were recorded in voltage-clamp mode at a holding potential of $-60 \mathrm{mV}$, which was less negative than the reversal potential for sIPSC. Indeed, for this set of experiments, we used the pipette solution contained a low concentration of $\mathrm{Cl}^{-}(1 \mathrm{mM})$, resulting in an $\mathrm{E}_{\mathrm{Cl}}$ of $-111 \mathrm{mV}$. Therefore, inward currents were considered as sEPSCs and outward currents as SIPSCs. Data were filtered at $2 \mathrm{kHz}$, digitized at $10 \mathrm{kHz}$ and acquired on-line using the pCLAMP 9 (Clampex) software (Axon Instruments). The recorded currents were subsequently analyzed using the Mini Analysis program (version 6.0.7; Synaposoft, Inc). The threshold for sIPSCs and sEPSCs detection was set at $7 \mathrm{pA}$, and the automatic detection was verified post hoc by visual inspection.

Statistics. All data are expressed as the mean \pm standard error of the mean (SEM). Statistical analyses were performed using Statistica software (Statsoft). Differences between clusters were analyzed by one-way analysis of variance (ANOVA), followed by a Newman-Keuls multiple comparison test. Differences between two neuronal populations were analyzed using a MannWhitney $U$-test. $P$-values of $\leq 0.05$ were considered statistically significant. In all cases, $\mathrm{n}$ refers to the number of examined neurons. 


\section{RESULTS}

\section{Unsupervised classification of VLPO neurons}

Two hundred eighty nine neurons were recorded from acute preoptic hypothalamic slices in the VLPO. Neurons were first electrophysiologically characterized by patch-clamp recordings (see Materials and Methods). The spontaneous discharge was assessed in cell-attached configuration and the 38 others discriminative electrophysiological features were measured for each cell from responses to hyperpolarizing and depolarizing current pulses in whole-cell current-clamp mode (see Materials and Methods). 18 principal components were selected from a principal component analysis. Based on these parameters, we applied the unsupervised clustering Ward's method (Ward 1963) to avoid a personal bias of determination and to group cells with large similarities into distinct subpopulations. A clustering tree was built, representing individual cells from its leaves and grouping them into the branched ramification up to a common root (Fig. 1A).

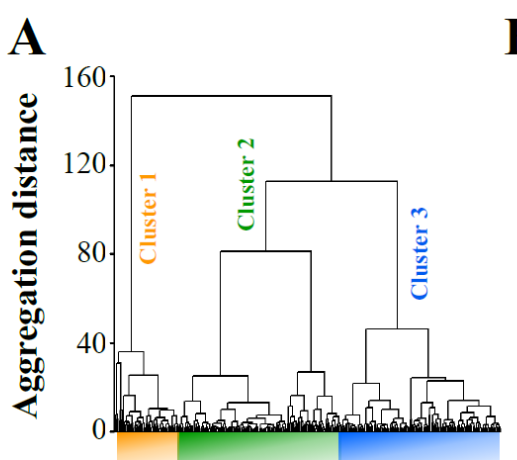

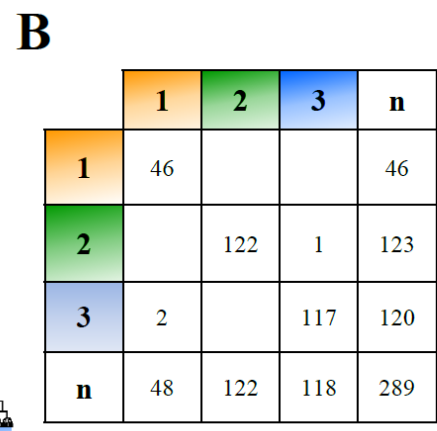

Ward clusters
C

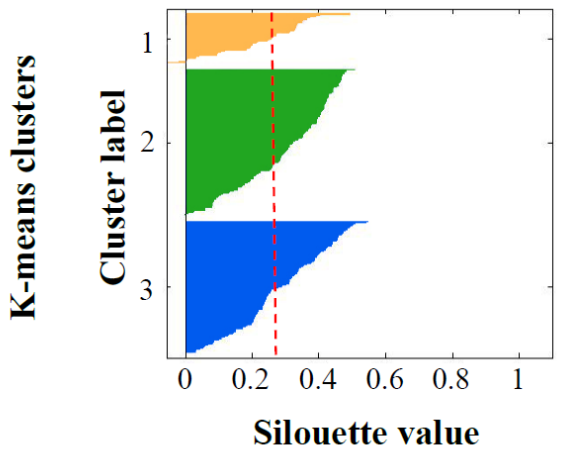

Fig. 1. Unsupervised clustering of VLPO neurons based on their electrophysiological properties. A, Ward's clustering of 289 VLPO neurons. Individual cells are represented along the $\mathrm{x}$-axis. The $\mathrm{y}$-axis represents the average within-cluster linkage distance in a space of 18 electrophysiological variables. Three clusters, 1 (in yellow), 2 (in green) and 3 (in blue) were identified. B, Clusters generated by Ward's method in (A) were corrected using the clustering output generated by the K-means algorithm. C, The silhouette analysis was used to assess the 
quality of the clustering that was optimal for 3 clusters with a mean value of 0.275 (red dash line).

The reliability of Ward's clustering was corrected using the K-mean algorithm. Two cells of cluster 1 were reassigned to cluster 3 , and one cell of cluster 3 was reassigned to cluster 2

(Fig. 1B). In order to determine whether the allocation of neurons into three groups was the optimal outcome of the clustering process, our results were compared with clustering trials whereby thresholds were set to define fewer or higher numbers of groups. We established that a cluster analysis yielding three groups was the best outcome with a mean silhouette value of 0.275 (Fig. 1C). Similarly, we applied the clustering Ward's method on two separate groups of neurons (163 and 126 neurons respectively). These unsupervised classifications also determined 3 clusters endowed with the same proportion of neurons than observed in Figure 1A. $95.09 \%$ out of the 163 neurons and $92.06 \%$ out of the 126 neurons segregated in the same cluster than in Figure 1A. Thus, the subdivision of VLPO neurons into three main groups was retained for further analysis. However, it appeared from the dendrogram that neurons from cluster 2 and 3 were likely composed of 2 distinct subgroups that were named $2 \mathrm{a}, 2 \mathrm{~b}$ and $3 \mathrm{a}, 3 \mathrm{~b}$ respectively. Therefore, we further analyzed the electrophysiological, morphological and pharmacological features of clusters $1,2 \mathrm{a}, 2 \mathrm{~b}, 3 \mathrm{a}$ and $3 \mathrm{~b}$ to define their properties.

\section{Electrophysiological diversity of VLPO neurons}

To characterize the neuronal diversity of VLPO neurons, the 39 discriminative electrophysiological features measured on our 289 cells were compared among the five identified clusters. Electrophysiological data regarding subthreshold properties among VLPO neurons showed that cluster $3 \mathrm{~b}$ neurons in particular display several distinctive features. They were characterized by a significantly higher input resistance (2) and an important Ih current, 
leading to higher slopes of I-V plots $(5,6)$ and a smaller sag index (7) compared to other groups (Table 1). Neurons from cluster $2 \mathrm{~b}$ were also characterized by their highest membrane capacitance (4).

Table 1. Comparison of passive membrane properties among VLPO neuron subtypes

\begin{tabular}{lccccc}
\hline & $\begin{array}{c}\text { Cluster 1 } \\
(n=46)\end{array}$ & $\begin{array}{c}\text { Cluster 2a } \\
(n=85)\end{array}$ & $\begin{array}{c}\text { Cluster 2b } \\
(n=38)\end{array}$ & $\begin{array}{c}\text { Cluster 3a } \\
(n=52)\end{array}$ & $\begin{array}{c}\text { Cluster 3b } \\
(n=68)\end{array}$ \\
\hline$(1) \mathbf{R M P}(\mathrm{mV})$ & $-40.0 \pm 1.1$ & $-44.2 \pm 0.7$ & $-41.9 \pm 0.7$ & $-42.7 \pm 0.7$ & $-42.2 \pm 0.6$ \\
& - & - & - & - & - \\
(2) $\mathbf{R}_{\mathbf{m}}(\mathrm{M} \Omega)$ & $557.9 \pm 42.1$ & $585.1 \pm 29.8$ & $626.5 \pm 38.7$ & $626.9 \pm 24.1$ & $991.3 \pm 42.5$ \\
& $1<<<3 \mathrm{~b}$ & $2 \mathrm{a}<<<3 \mathrm{~b}$ & $2 \mathrm{~b}<<<3 \mathrm{~b}$ & $3 \mathrm{a}<<<3 \mathrm{~b}$ & $3 \mathrm{~b}>>>1,2 \mathrm{a}, 2 \mathrm{~b}, 3 \mathrm{a}$ \\
& $32.9 \pm 2.5$ & $31.3 \pm 2.1$ & $41.6 \pm 1.8$ & $27.9 \pm 1.3$ & $41.4 \pm 1.7$ \\
(3) $\boldsymbol{\tau}_{\mathbf{m}}(\mathrm{ms})$ & $1<<2 \mathrm{~b}$ & $2 \mathrm{a}<<<2 \mathrm{~b}, 3 \mathrm{~b}$ & $2 \mathrm{~b}>1>>>2 \mathrm{a}, 3 \mathrm{a}$ & $3 \mathrm{a}<<<2 \mathrm{~b}, 3 \mathrm{~b}$ & $3 \mathrm{~b}>>>2 \mathrm{a}, 3 \mathrm{a}$ \\
& $63.6 \pm 3.7$ & $56.7 \pm 2.5$ & $86.3 \pm 4.4$ & $47.4 \pm 2.0$ & $45.7 \pm 1.7$ \\
(4) $\mathbf{C}_{\mathbf{m}}(\mathrm{pF})$ & $3 \mathrm{~b}<<\mathrm{a}<1<<<2 \mathrm{~b}$ & $2 \mathrm{a}<<<2 \mathrm{~b}$ & $2 \mathrm{~b}>>>>1,2 \mathrm{a}, 3 \mathrm{a}, 3 \mathrm{~b}$ & $3 \mathrm{a}<1<<<2 \mathrm{~b}$ & $3 \mathrm{~b}<<1<<<2 \mathrm{~b}$ \\
& $389.7 \pm 30.7$ & $383.0 \pm 17.7$ & $480.5 \pm 28.8$ & $444.7 \pm 17.0$ & $700.4 \pm 25.7$ \\
(5) $\mathbf{G}_{\mathbf{h y p}}(\mathrm{M} \Omega)$ & $1<<<3 \mathrm{~b}$ & $2 \mathrm{a}<<<3 \mathrm{~b}$ & $2 \mathrm{~b}<<<3 \mathrm{~b}$ & $3 \mathrm{a}<<<3 \mathrm{~b}$ & $3 \mathrm{~b}>>>1,2 \mathrm{a}, 2 \mathrm{~b}, 3 \mathrm{a}$ \\
& $290.3 \pm 21.5$ & $311.6 \pm 13.4$ & $359.1 \pm 17.3$ & $362.2 \pm 12.2$ & $427.5 \pm 16.2$ \\
(6) $\mathbf{G}_{\mathbf{s a g}}(\mathrm{M} \Omega)$ & $1<<<3 \mathrm{~b}$ & $2 \mathrm{a}<<<3 \mathrm{~b}$ & $2 \mathrm{~b}<3 \mathrm{~b}$ & $3 \mathrm{~b}>3 \mathrm{a}$ & $3 \mathrm{~b}>2 \mathrm{~b}, 3 \mathrm{a}>>>1,2 \mathrm{a}$ \\
& $76.8 \pm 2.1$ & $83.8 \pm 1.2$ & $80.4 \pm 1.3$ & $82.7 \pm 0.8$ & $63.5 \pm 1.6$ \\
(7) $\Delta \mathbf{G}_{\mathbf{s a g}}(\%)$ & $3 \mathrm{~b}<<<1<2 \mathrm{a}$ & $2 \mathrm{a}>1>>>3 \mathrm{~b}$ & $2 \mathrm{~b}>>>3 \mathrm{~b}$ & $3 \mathrm{a}>>>3 \mathrm{~b}$ & $3 \mathrm{~b}<<<1,2 \mathrm{a}, 2 \mathrm{~b}, 3 \mathrm{a}$ \\
\hline
\end{tabular}

$n$, Number of cells; < indicates significantly smaller with $P \leq 0.05 ;<<$ indicates significantly smaller with $P \leq 0.01 ;<<<$ indicates significantly smaller with $P \leq 0.001$; one-way ANOVA, followed by a Newman-Keuls test. Values significantly different in one cluster $v s$. all others were highlighted in gray.

When depolarized just above threshold, neurons from cluster $3 \mathrm{~b}$ significantly exhibited a lower rheobase (9), whereas neurons from cluster $2 \mathrm{~b}$ displayed significant longer first spike latency (10), compared to other neurons (Table 2). Neurons from cluster 1 were also distinguished from other groups by their higher adaptation (11) and steady state firing frequency (12) at threshold currents (Table 2). 
Table 2. Comparison of just above threshold properties among VLPO neuron subtypes

\begin{tabular}{lccccc}
\hline & $\begin{array}{c}\text { Cluster 1 } \\
(n=46)\end{array}$ & $\begin{array}{c}\text { Cluster 2a } \\
(n=85)\end{array}$ & $\begin{array}{c}\text { Cluster 2b } \\
(n=38)\end{array}$ & $\begin{array}{c}\text { Cluster 3a } \\
(n=52)\end{array}$ & $\begin{array}{c}\text { Cluster 3b } \\
(n=68)\end{array}$ \\
\hline (8) $\mathbf{A P}$ th $(\mathrm{mV})$ & $-40.5 \pm 0.7$ & $-39.8 \pm 0.4$ & $-40.1 \pm 0.6$ & $-42.4 \pm 0.5$ & $-43.7 \pm 0.4$ \\
& - & - & - & - & - \\
& $22.4 \pm 2.0$ & $30.0 \pm 1.5$ & $24.9 \pm 2.0$ & $18.5 \pm 1.0$ & $11.9 \pm 0.5$ \\
(9) Rheobase (pA) & $3 \mathrm{~b}<<<1<2 \mathrm{a}$ & $2 \mathrm{a}>1>>>3 \mathrm{a}, 3 \mathrm{~b}$ & $3 \mathrm{~b}<<<2 \mathrm{~b}$ & $3 \mathrm{~b}<3 \mathrm{a}<<<2 \mathrm{a}$ & $3 \mathrm{~b}<3 \mathrm{a}<<<1,2 \mathrm{a}, 2 \mathrm{~b}$ \\
& $96.2 \pm 7.9$ & $113.6 \pm 5.7$ & $287.4 \pm 17.5$ & $88.7 \pm 5.1$ & $86.8 \pm 5.7$ \\
$(10) \mathbf{1}^{\text {st }}$ spike (ms) & $1<<<2 \mathrm{~b}$ & $2 \mathrm{a}<<<2 \mathrm{~b}$ & $2 \mathrm{~b}>>>1,2 \mathrm{a}, 3 \mathrm{a}, 3 \mathrm{~b}$ & $3 \mathrm{a}<<<2 \mathrm{~b}$ & $3 \mathrm{~b}<<<2 \mathrm{~b}$ \\
& $-274.0 \pm 105.0$ & $-9.2 \pm 2.2$ & $-5.8 \pm 1.8$ & $-11.7 \pm 1.6$ & $-8.5 \pm 1.2$ \\
& $1<<<2 \mathrm{a}, 2 \mathrm{~b}, 3 \mathrm{a}, 3 \mathrm{~b}$ & $2 \mathrm{a}<<<1$ & $2 \mathrm{~b}<<<1$ & $3 \mathrm{a}<<<1$ & $3 \mathrm{~b}<<<1$ \\
(11) $\mathbf{m}_{\text {th }}(\mathrm{Hz} / \mathrm{s})$ & $50.2 \pm 4.6$ & $7.6 \pm 0.4$ & $8.2 \pm 0.7$ & $11.0 \pm 0.6$ & $12.2 \pm 11.0$ \\
& $1>>>2 \mathrm{a}, 2 \mathrm{~b}, 3 \mathrm{a}, 3 \mathrm{~b}$ & $2 \mathrm{a}<<<1$ & $2 \mathrm{~b}<<<1$ & $3 \mathrm{a}<<<<1$ & $3 \mathrm{~b}<<<<1$ \\
\hline
\end{tabular}

$n$, Number of cells; < indicates significantly smaller with $P \leq 0.05 ;<<$ indicates significantly smaller with $P \leq 0.01 ;<<<$ indicates significantly smaller with $P \leq 0.001$; one-way ANOVA, followed by a Newman-Keuls test. Values significantly different in one cluster $v \boldsymbol{s}$. all others were highlighted in gray.

A

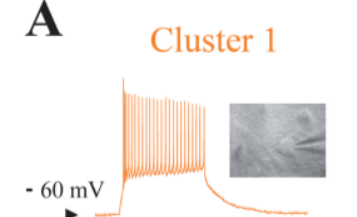

$\rightarrow$

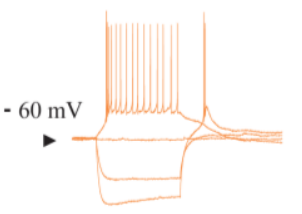

B

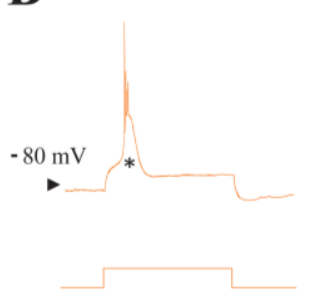

Cluster $2 \mathrm{a}$
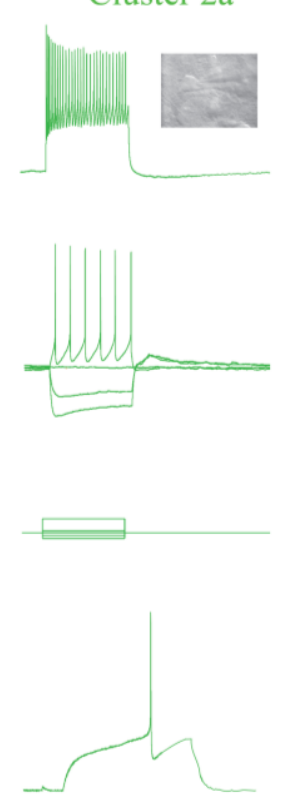

Cluster $2 \mathrm{~b}$
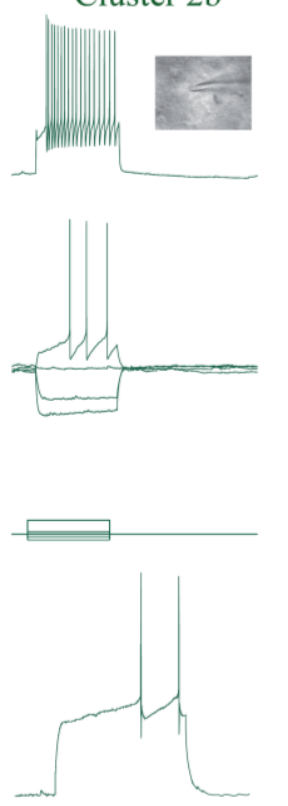

Cluster $3 \mathrm{a}$
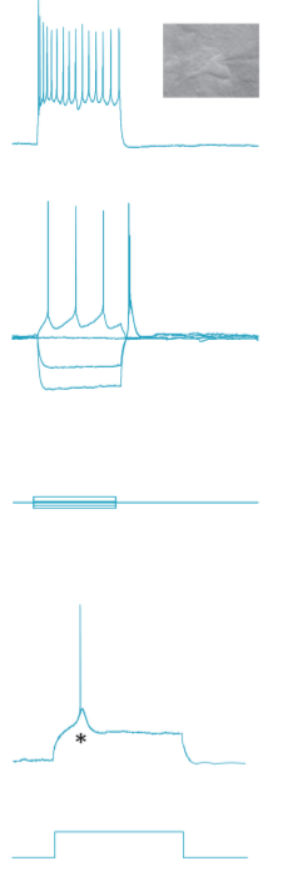

Cluster $3 b$
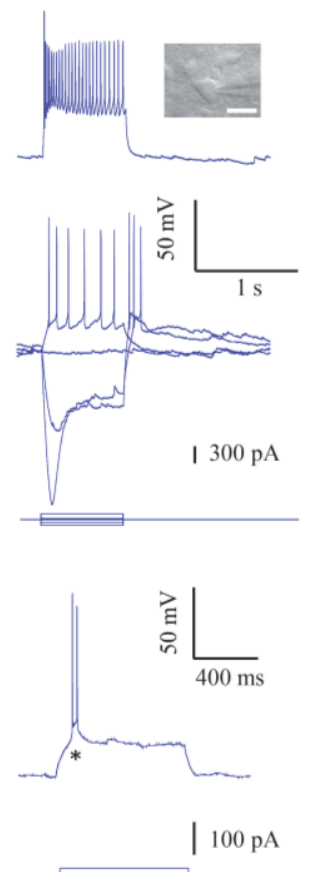

Fig. 2. Electrophysiological properties of VLPO neurons recorded in current-clamp. A, Typical electrophysiological behavior of neurons from each cluster. Neurons from cluster 1 (yellow), 2a (light green), $2 \mathrm{~b}$ (dark green), $3 \mathrm{a}$ (light blue) and $3 \mathrm{~b}$ (dark blue) were recorded using wholecell patch-clamp recordings in response to the application of current pulses (bottom traces). Strong depolarizing current (top trace) evoked a high and sustained firing in all clusters. Top insets, IR pictures of the recorded neurons (scale bar, $20 \mu \mathrm{m}$ ). B, Characterization of LTS and 
non-LTS VLPO neurons. Current-clamp recordings of typical neurons from all clusters are represented at their spiking threshold when depolarized from a hyperpolarized level. Cluster 1 , $3 \mathrm{a}$ and $3 \mathrm{~b}$ cells display an LTS (asterisk). Cluster $2 \mathrm{a}$ and $2 \mathrm{~b}$ non-LTS cells show a voltage response towards the first action potential and no LTS. These neuronal properties were recorded in response to the application of current pulses (bottom traces).

VLPO neurons also exhibited a wide diversity of evoked near threshold firing patterns (Fig. 2). Burst firing neurons (14) were essentially found in cluster 1, non fast-spiking non-adapting firing neurons (15) in cluster $3 \mathrm{~b}$, adapting firing neurons (16) in cluster $2 \mathrm{a}$, late spiking firing neurons (17) in cluster $2 \mathrm{~b}$ and irregular firing neurons (18) in cluster 3a (Table 3). However, clusters $2 \mathrm{a}$ and $3 \mathrm{a}$ were also endowed with a high proportion of non fast-spiking non-adapting firing neurons.

Table 3. Comparison of firing properties among VLPO neuronal subtypes

\begin{tabular}{|c|c|c|c|c|c|}
\hline & $\begin{array}{c}\text { Cluster 1 } \\
(n=46)\end{array}$ & $\begin{array}{c}\text { Cluster 2a } \\
(n=85)\end{array}$ & $\begin{array}{c}\text { Cluster 2b } \\
(n=38)\end{array}$ & $\begin{array}{c}\text { Cluster 3a } \\
(n=52)\end{array}$ & $\begin{array}{c}\text { Cluster 3b } \\
(n=68)\end{array}$ \\
\hline (13) Spont. (Hz) & $\begin{array}{c}3.6 \pm 0.7 \\
-\end{array}$ & $\begin{array}{c}1.9 \pm 0.3 \\
2 \mathrm{a}<3 \mathrm{~b}\end{array}$ & $\begin{array}{c}2.7 \pm 0.2 \\
2 b<3 b\end{array}$ & $\begin{array}{c}3.8 \pm 0.3 \\
-\end{array}$ & $\begin{array}{l}4.6 \pm 0.3 \\
3 b>2 a, 2 b\end{array}$ \\
\hline (14) Burst (\%) & $\begin{array}{c}97.8 \\
1>>2 \mathrm{a}, 2 \mathrm{~b}, 3 \mathrm{a}, 3 \mathrm{~b}\end{array}$ & $\begin{array}{c}0 \\
2 \mathrm{a}<<<1\end{array}$ & $\begin{array}{c}0 \\
2 b<<<1\end{array}$ & $\begin{array}{c}11.5 \\
3 \mathrm{a}<<<1\end{array}$ & $\begin{array}{c}4.4 \\
3 b<<<1\end{array}$ \\
\hline $\begin{array}{l}\text { (15) Non fast spiking } \\
\text { non adapting }(\%)\end{array}$ & $\frac{0}{1<<<2 \mathrm{a}, 3 \mathrm{a}, 3 \mathrm{~b}}$ & $\begin{array}{c}51.8 \\
3 \mathrm{~b} \gg>>2 \mathrm{a} \gg>>1,2 \mathrm{~b}\end{array}$ & $\begin{array}{c}2.6 \\
2 \mathrm{~b}<<<2 \mathrm{a}, 3 \mathrm{a}, 3 \mathrm{~b}\end{array}$ & $\begin{array}{c}48.1 \\
1,2 b<<<3 a<<<3 b\end{array}$ & $\begin{array}{c}86.8 \\
3 \mathrm{~b} \gg>>1,2 \mathrm{a}, 2 \mathrm{~b}, 3 \mathrm{a}\end{array}$ \\
\hline (16) Adpating (\%) & $\begin{array}{c}0 \\
1<<3 a<<<2 a, 3 b\end{array}$ & $\begin{array}{c}40.0 \\
2 a>3 a \gg \gg 1,2 b, 3 b\end{array}$ & $\begin{array}{c}0 \\
1<<2 b<<<2 a, 3 b\end{array}$ & $\begin{array}{c}23.1 \\
2 a>3 a>1,3 a, 3 b\end{array}$ & $\begin{array}{c}4.4 \\
3 b<<3 a<<<2 a\end{array}$ \\
\hline (17) Late spiking (\%) & $\begin{array}{l}2.2 \\
1<<<2 b\end{array}$ & $\begin{array}{c}0 \\
2 \mathrm{a}<<<2 \mathrm{~b}\end{array}$ & $\begin{array}{c}97.4 \\
2 \mathrm{~b}>>>1,2 \mathrm{a}, 3 \mathrm{a}, 3 \mathrm{~b}\end{array}$ & $\begin{array}{c}0 \\
3 a<<<2 b\end{array}$ & $\begin{array}{c}1.5 \\
3 b<<<2 b\end{array}$ \\
\hline (18) Irregular (\%) & $\begin{array}{c}0 \\
1<<<3 a\end{array}$ & $\begin{array}{c}8.2 \\
2 a<3 a\end{array}$ & $\begin{array}{c}0 \\
2 b<<3 a\end{array}$ & $\begin{array}{c}17.3 \\
3 a>2 a>>2 b, 3 b>>> \\
1\end{array}$ & $\begin{array}{c}2.9 \\
3 \mathrm{~b}<<3 \mathrm{a}\end{array}$ \\
\hline
\end{tabular}

$n$, Number of cells; < indicates significantly smaller with $P \leq 0.05 ;<<$ indicates significantly smaller with $P \leq 0.01 ;<<<$ indicates significantly smaller with $P \leq 0.001$; one-way ANOVA followed by a Newman-Keuls test . Values significantly different in one cluster $v s$. all others were highlighted in gray.

Comparison of action potential properties among VLPO neurons essentially revealed that neurons from cluster 1 exhibit significant smaller AHP amplitude (21) and duration (22), a reduced second action potential amplitude (23, Table 4). Accordingly, they displayed a higher 
action potential amplitude variation (25) and duration (26). At higher stimulation intensities, they also presented a higher amplitude of early adaptation (27; Table 4).

Table 4. Comparison of action potential properties among VLPO neurons

\begin{tabular}{|c|c|c|c|c|c|}
\hline & $\begin{array}{c}\text { Cluster 1 } \\
(n=46)\end{array}$ & $\begin{array}{c}\text { Cluster 2a } \\
(n=85)\end{array}$ & $\begin{array}{c}\text { Cluster 2 b } \\
(n=38)\end{array}$ & $\begin{array}{c}\text { Cluster 3a } \\
(n=52)\end{array}$ & $\begin{array}{c}\text { Cluster } 3 \mathbf{b} \\
(n=68)\end{array}$ \\
\hline (19) A1 (mV) & $\begin{array}{c}89.3 \pm 2.6 \\
-\end{array}$ & $\begin{array}{c}91.0 \pm 1.5 \\
-\end{array}$ & $\begin{array}{c}91.8 \pm 1.2 \\
-\end{array}$ & $\begin{array}{c}97.4 \pm 1.8 \\
-\end{array}$ & $\begin{array}{c}90.5 \pm 1.2 \\
-\end{array}$ \\
\hline (20) D1 (ms) & $1.1 \pm 0.0$ & $1.1 \pm 0.0$ & $\begin{array}{c}1.2 \pm 0.0 \\
-\end{array}$ & $1.1 \pm 0.0$ & $\begin{array}{c}1.2 \pm 0.0 \\
-\end{array}$ \\
\hline $\begin{array}{l}\text { (21) } \text { AHP }_{\max } \\
\text { amplitude (mV) }\end{array}$ & $\begin{array}{c}-1.4 \pm 0.3 \\
1<<<2 \mathrm{a}, 2 \mathrm{~b}, 3 \mathrm{a}, 3 \mathrm{~b}\end{array}$ & $\begin{array}{c}-10.1 \pm 0.4 \\
2 a>>>1,3 a\end{array}$ & $\begin{array}{c}-10.2 \pm 0.6 \\
2 \mathrm{~b} \gg>>1,3 \mathrm{a}\end{array}$ & $\begin{array}{c}-5.2 \pm 0.3 \\
2 b, 3 b \gg>>3 a>>>1\end{array}$ & $\begin{array}{l}-8.9 \pm 0.4 \\
3 b>>>1,3 a\end{array}$ \\
\hline $\begin{array}{l}\text { (22) } \text { AHP }_{\max } \\
\text { duration }(\mathrm{ms})\end{array}$ & $\begin{array}{c}6.1 \pm 3.1 \\
1<<<2 \mathrm{a}, 2 \mathrm{~b}, 3 \mathrm{a}, 3 \mathrm{~b}\end{array}$ & $\begin{array}{l}131.9 \pm 5.5 \\
2 \mathrm{a}>>>1,3 \mathrm{a}, 3 \mathrm{~b}\end{array}$ & $\begin{array}{l}130.7 \pm 5.8 \\
2 \mathrm{~b} \gg>1,3 \mathrm{a}, 3 \mathrm{~b}\end{array}$ & $\begin{array}{c}99.5 \pm 6.7 \\
2 a, 2 b \gg>>3 a \gg>1\end{array}$ & $\begin{array}{c}90.8 \pm 5.5 \\
2 \mathrm{a}, 2 \mathrm{~b} \gg \gg>3 \mathrm{~b} \gg>>1\end{array}$ \\
\hline (23) $\mathbf{A} \mathbf{2}(\mathrm{mV})$ & $69.8 \pm 2.6$ & $85.3 \pm 1.3$ & $88.1 \pm 1.3$ & $89.3 \pm 1.8$ & $85.1 \pm 1.1$ \\
\hline (24) D2 (ms) & $\begin{array}{c}1<<<2 \mathrm{a}, 2 \mathrm{~b}, 3 \mathrm{a}, 3 \mathrm{~b} \\
1.4 \pm 0.1 \\
1>2 \mathrm{a}, 3 \mathrm{a}\end{array}$ & $\begin{array}{c}2 \mathrm{a}>>>1 \\
1.2 \pm 0.0 \\
2 \mathrm{a}<1\end{array}$ & $\begin{array}{c}2 \mathrm{~b} \gg>>1 \\
1.2 \pm 0.0 \\
-\end{array}$ & $\begin{array}{c}3 a>>>1 \\
1.2 \pm 0.0 \\
3 a<1\end{array}$ & $\begin{array}{c}3 b>>>1 \\
1.3 \pm 0.0 \\
-\end{array}$ \\
\hline$(25) \operatorname{Var} \mathbf{A}(\%)$ & $\begin{array}{c}22.0 \pm 1.8 \\
1 \gg>2 \mathrm{a}, 2 \mathrm{~b}, 3 \mathrm{a}, 3 \mathrm{~b}\end{array}$ & $\begin{array}{c}6.1 \pm 0.4 \\
2 a \gg>>1\end{array}$ & $\begin{array}{c}3.9 \pm 0.5 \\
3 b \gg>>1\end{array}$ & $\begin{array}{c}8.4 \pm 0.6 \\
3 a \gg>>1\end{array}$ & $\begin{array}{c}6.0 \pm 0.4 \\
3 b \gg>1\end{array}$ \\
\hline (26) Var D (\%) & $\begin{array}{c}-24.5 \pm 3.8 \\
1 \gg \gg 2 \mathrm{a}, 2 \mathrm{~b}, 3 \mathrm{a}, 3 \mathrm{~b}\end{array}$ & $\begin{array}{c}-7.0 \pm 0.6 \\
2 a<<<1\end{array}$ & $\begin{array}{c}-5.9 \pm 0.5 \\
2 b<<<1\end{array}$ & $\begin{array}{c}-8.4 \pm 0.8 \\
3 a<<<1\end{array}$ & $\begin{array}{c}-6.2 \pm 0.6 \\
3 b<<<1\end{array}$ \\
\hline (27) $\mathbf{A}_{\text {sat }}(\mathrm{Hz})$ & $\begin{array}{c}92.2 \pm 5.9 \\
1>>2 \mathrm{a}, 2 \mathrm{~b}, 3 \mathrm{a}, 3 \mathrm{~b}\end{array}$ & $\begin{array}{l}62.2 \pm 5.0 \\
2 \mathrm{~b}<2 \mathrm{a}<<<1\end{array}$ & $\begin{array}{c}39.0 \pm 4.3 \\
2 b<2 a 3 a<<<1\end{array}$ & $\begin{array}{l}61.9 \pm 2.3 \\
2 b<3 a<<<1\end{array}$ & $\begin{array}{c}54.5 \pm 3.1 \\
3 \mathrm{~b}<<<1\end{array}$ \\
\hline (28) $\tau_{\text {sat }}(\mathrm{ms})$ & $\begin{array}{c}26.0 \pm 2.6 \\
-\end{array}$ & $\begin{array}{c}43.2 \pm 6.2 \\
-\end{array}$ & $\begin{array}{c}37.3 \pm 4.6 \\
-\end{array}$ & $\begin{array}{c}28.0 \pm 1.5 \\
-\end{array}$ & $\begin{array}{c}51.7 \pm 7.1 \\
-\end{array}$ \\
\hline (29) $\mathbf{F}_{\max }(\mathrm{Hz})$ & $\begin{array}{c}44.8 \pm 5.2 \\
-\end{array}$ & $\begin{array}{c}48.0 \pm 2.6 \\
-\end{array}$ & $\begin{array}{c}59.9 \pm 5.6 \\
-\end{array}$ & $\begin{array}{c}48.8 \pm 2.9 \\
-\end{array}$ & $\begin{array}{c}58.9 \pm 3.2 \\
-\end{array}$ \\
\hline (30) $\mathbf{m}_{\mathrm{sat}}(\mathrm{Hz} / \mathrm{s})$ & $-18.9 \pm 2.2$ & $-15.6 \pm 1.70$ & $-22.4 \pm 2.6$ & $\begin{array}{c}-5.6 \pm 5.9 \\
-\end{array}$ & $\begin{array}{c}-17.2 \pm 1.3 \\
-\end{array}$ \\
\hline
\end{tabular}

$n$, Number of cells; < indicates significantly smaller with $P \leq 0.05 ;<<$ indicates significantly smaller with $P \leq 0.01 ;<<$ indicates significantly smaller with $P \leq 0.001$; one-way ANOVA followed by a Newman-Keuls test . Values significantly different in one cluster $v s$. all others were highlighted in gray.

The presence of an LTS is one of the electrophysiological landmark of sleep-promoting neurons (Gallopin et al. 2000; Matsuo et al. 2003; Gallopin et al. 2004; Saint-Mleux et al. 2004; SaintMleux et al. 2007; Liu et al. 2010; Moore et al. 2012) that was frequently observed in clusters 1 and 3 (31; Table 5 and Fig. 2). However, we observed no statistical difference regarding LTS properties among VLPO clusters. An outward rectification (35) appeared to be a specific feature of cluster 2 neurons (Table 5). 
Table 5. Comparison of LTS properties among VLPO neurons

\begin{tabular}{lccccc}
\hline & $\begin{array}{c}\text { Cluster 1 } \\
(n=37)\end{array}$ & $\begin{array}{c}\text { Cluster 2a } \\
(n=4)\end{array}$ & $\begin{array}{c}\text { Cluster 2b } \\
(n=8)\end{array}$ & $\begin{array}{c}\text { Cluster 3a } \\
(n=47)\end{array}$ & $\begin{array}{c}\text { Cluster 3b } \\
(n=54)\end{array}$ \\
\hline (31) LTS (\%) & 80.4 & 4.7 & 21.1 & 90.4 & 79.4 \\
& $1>>>2 \mathrm{a}, 2 \mathrm{~b}$ & $2 \mathrm{a}<<2 \mathrm{~b}<<<1,3 \mathrm{a}, 3 \mathrm{~b}$ & $2 \mathrm{a}<<2 \mathrm{~b}<<<1,3 \mathrm{a}, 3 \mathrm{~b}$ & $3 \mathrm{a}>>>2 \mathrm{a}, 2 \mathrm{~b}$ & $3 \mathrm{~b}>>>2 \mathrm{a}, 2 \mathrm{~b}$ \\
(32) LTS & $19.7 \pm 1.3$ & $13.8 \pm 0.4$ & $11.0 \pm 2.7$ & $15.8 \pm 1.1$ & $14.8 \pm 1.0$ \\
amplitude (mV) & - & - & - & - & - \\
(33) LTS & $178.6 \pm 14.9$ & $200.8 \pm 37.8$ & $213.9 \pm 48.5$ & $187.0 \pm 12.1$ & $225.9 \pm 21.0$ \\
duration (ms) & - & - & - & - & - \\
(34) AP/burst & $3.1 \pm 0.4$ & $1.2 \pm 0.0$ & $1.5 \pm 0.3$ & $1.9 \pm 0.2$ & $2.4 \pm 0.2$ \\
& - & - & - & - & - \\
(35) Slope (\%) & $0.2 \pm 0.2$ & $1.3 \pm 0.4$ & $3.7 \pm 1.5$ & $0.0 \pm 0.0$ & $0.0 \pm 0.0$ \\
& $1<<<<\mathrm{b}$ & $2 \mathrm{a}<2 \mathrm{~b}$ & $2 \mathrm{~b}>2 \mathrm{a}>>>1,3 \mathrm{a}, 3 \mathrm{~b}$ & $3 \mathrm{a}<<<2 \mathrm{~b}$ & $3 \mathrm{~b}<<<<2 \mathrm{~b}$ \\
\hline
\end{tabular}

$n$, Number of cells; < indicates significantly smaller with $P \leq 0.05 ;<<$ indicates significantly smaller with $P \leq 0.01 ;<<<$ indicates significantly smaller with $P \leq 0.001$; one-way ANOVA, followed by a Newman-Keuls test. Values significantly different in one cluster $v s$. all others were highlighted in gray.

The post-inhibitory rebound following hyperpolarizing current from $-60 \mathrm{mV}$ was frequently recorded and also represents a discriminative parameter of VLPO neurons (Table 6). In particular, cluster 2 neurons were significantly less frequently endowed with a post-inhibitory rebound (36) than neurons from clusters 1 and 3. Post-inhibitory rebounds also had distinct kinetics among the VLPO neuronal clusters: the mean amplitude (37) was significantly higher in cluster 1 vs. the other clusters, and lasted longer (38) in the cluster $3 \mathrm{~b}$ (Table 6). Finally, we found that neurons from cluster $3 \mathrm{~b}$ fired significantly more action potential per rebound than neurons from clusters $2 \mathrm{a}, 2 \mathrm{~b}$ and $3 \mathrm{a}$ (39; Table 6).

Table 6. Comparison of overshoot of the membrane potential properties among VLPO neurons

\begin{tabular}{|c|c|c|c|c|c|}
\hline & $\begin{array}{c}\text { Cluster 1 } \\
(n=37)\end{array}$ & $\begin{array}{c}\text { Cluster 2a } \\
(n=15) \\
\end{array}$ & $\begin{array}{c}\text { Cluster 2b } \\
(n=19)\end{array}$ & $\begin{array}{c}\text { Cluster 3a } \\
(n=42)\end{array}$ & $\begin{array}{c}\text { Cluster 3b } \\
(n=66) \\
\end{array}$ \\
\hline (36) Rebound (\%) & 80.4 & 17.7 & 50.0 & 80.8 & 97.1 \\
\hline \multirow{2}{*}{$\begin{array}{l}\text { (37) Rebound } \\
\text { amplitude (mV) }\end{array}$} & $\begin{array}{c}3 \mathrm{~b}>>>1>>>2 \mathrm{a}, 2 \mathrm{~b} \\
22.5 \pm 1.2\end{array}$ & $\begin{array}{c}2 \mathrm{a}<<<1,2 \mathrm{~b}, 3 \mathrm{a}, 3 \mathrm{~b} \\
12.5 \pm 0.5\end{array}$ & $\begin{array}{c}2 \mathrm{a}<<<2 \mathrm{~b}<<<1,3 \mathrm{a}, 3 \mathrm{~b} \\
11.8 \pm 0.5\end{array}$ & $\begin{array}{c}3 \mathrm{~b}>3 \mathrm{a} \mathrm{p}>>>2 \mathrm{a}, 2 \mathrm{~b} \\
17.4 \pm 0.7\end{array}$ & $\begin{array}{c}3 \mathrm{~b}>3 \mathrm{a}>>>1,2 \mathrm{a}, 2 \mathrm{~b} \\
16.0 \pm 0.6\end{array}$ \\
\hline & $\begin{array}{c}1>3 \mathrm{a}>>3 \mathrm{~b}>>>2 \mathrm{a}, 2 \\
\mathrm{~b}\end{array}$ & $2 a<3 a<<<1$ & $2 b<3 a<<<1$ & $2 a, 2 b<3 a<1$ & $3 b<<1$ \\
\hline (38) Rebound & $501.6 \pm 64.8$ & $264.0 \pm 27.0$ & $693.6 \pm 37.5$ & $365.0 \pm 24.2$ & $1022.1 \pm 45.4$ \\
\hline duration $(\mathrm{ms})$ & $3 b>>>1>>2 a$ & $2 a<<1,2 b<<<3 b$ & $3 b>>2 b>2 a>>3 a$ & $3 b>>>2 b>3 a$ & $3 \mathrm{~b} \gg>2 \mathrm{~b} \gg>>1,2 \mathrm{a}, 3 \mathrm{a}$ \\
\hline (39) AP/burst & $\begin{array}{c}4.1 \pm 0.50 \\
1 \gg>2 \mathrm{a}, 3 \mathrm{a}\end{array}$ & $\begin{array}{c}2.0 \pm 0.2 \\
2 a<<3 b\end{array}$ & $\begin{array}{c}3.0 \pm 0.2 \\
3 b>2 b\end{array}$ & $\begin{array}{c}2.4 \pm 0.1 \\
3 a<3 b\end{array}$ & $\begin{array}{c}6.4 \pm 0.7 \\
3 b>2 b, 3 a>>2 a\end{array}$ \\
\hline
\end{tabular}


$n$, Number of cells; < indicates significantly smaller with $P \leq 0.05 ;<<$ indicates significantly smaller with $P \leq 0.01 ;<<<$ indicates significantly smaller with $P \leq 0.001$; one-way ANOVA, followed by a Newman-Keuls test. Values significantly different in one cluster $v s$. all others were highlighted in gray.

\section{Morphological properties of VLPO neurons}

Basic morphometric analyses of somata were determined from infrared picture taken prior recording. Multipolar cells with round, polygonal and triangular somata as bipolar/fusiform neurons were found in relative equivalent proportion in all neuronal clusters. Yet, to avoid a subjective classification of somata shape, morphological parameters were extracted from their measurements. In particular, this analyze revealed that neurons from the cluster $2 \mathrm{~b}$ displayed a cell body perimeter significantly bigger than somata from other clusters (41; Table 7).

Table 7. Somatic properties among VLPO neurons

\begin{tabular}{|c|c|c|c|c|c|}
\hline & $\begin{array}{c}\text { Cluster 1 } \\
(n=46) \\
\end{array}$ & $\begin{array}{c}\text { Cluster 2a } \\
(n=85) \\
\end{array}$ & $\begin{array}{c}\text { Cluster 2b } \\
(n=38)\end{array}$ & $\begin{array}{c}\text { Cluster 3a } \\
(n=52) \\
\end{array}$ & $\begin{array}{c}\text { Cluster 3b } \\
(n=68) \\
\end{array}$ \\
\hline (40) Cell body area $\left(\mu \mathrm{m}^{2}\right)$ & $\begin{array}{c}174.6 \pm 8.7 \\
1<<<2 b\end{array}$ & $\begin{array}{c}192.9 \pm 8.4 \\
2<<2 b\end{array}$ & $\begin{array}{c}232 \pm 11.4 \\
2 \mathrm{~b}>>2 \mathrm{a} \gg>>1,3 \mathrm{a}\end{array}$ & $\begin{array}{c}173.6 \pm 7.7 \\
3 \mathrm{a}<<<2 \mathrm{~b}\end{array}$ & $\begin{array}{c}205.9 \pm 9.6 \\
-\end{array}$ \\
\hline (41) Perimeter $(\mu \mathrm{m})$ & $\begin{array}{c}54.4 \pm 1.9 \\
1<<2 b\end{array}$ & $\begin{array}{c}54.8 \pm 1.4 \\
2 \mathrm{a}<<2 \mathrm{~b}\end{array}$ & $\begin{array}{c}61.4 \pm 1.7 \\
2 b>3 b>>1,2 a, 3 a\end{array}$ & $\begin{array}{c}53.4 \pm 1.4 \\
3 \mathrm{a} a<2 \mathrm{~b}\end{array}$ & $\begin{array}{c}56.4 \pm 1.4 \\
3 \mathrm{~b}<2 \mathrm{~b}\end{array}$ \\
\hline (42) Form factor & $\begin{array}{c}0.8 \pm 0 \\
-\end{array}$ & $\begin{array}{c}0.8 \pm 0 \\
-\end{array}$ & $\begin{array}{c}0.8 \pm 0 \\
-\end{array}$ & $\begin{array}{c}0.8 \pm 0 \\
-\end{array}$ & $\begin{array}{c}0.8 \pm 0 \\
-\end{array}$ \\
\hline (43) Feret $\max (\mu \mathrm{m})$ & $\begin{array}{c}20.5 \pm 0.8 \\
-\end{array}$ & $\begin{array}{c}20.6 \pm 0.6 \\
-\end{array}$ & $\begin{array}{c}22.9 \pm 0.8 \\
-\end{array}$ & $\begin{array}{c}20.4 \pm 0.6 \\
-\end{array}$ & $\begin{array}{c}21.1 \pm 0.6 \\
-\end{array}$ \\
\hline (44) Feret min $(\mu \mathrm{m})$ & $\begin{array}{l}12.4 \pm 0.4 \\
1<3 b<<<2 b\end{array}$ & $\begin{array}{c}12.9 \pm 0.3 \\
2 \mathrm{a}<2 \mathrm{~b}\end{array}$ & $\begin{array}{c}14.5 \pm 0.5 \\
2 b>2 a>>>1,3 a\end{array}$ & $\begin{array}{c}12.3 \pm 0.3 \\
<<<2 \mathrm{~b}\end{array}$ & $\begin{array}{c}13.6 \pm 0.4 \\
-\end{array}$ \\
\hline (45) Aspect ratio & $\begin{array}{c}1.7 \pm 0.1 \\
-\end{array}$ & $\begin{array}{c}1.6 \pm 0 \\
-\end{array}$ & $\begin{array}{c}1.6 \pm 0.1 \\
-\end{array}$ & $\begin{array}{c}1.7 \pm 0.1 \\
-\end{array}$ & $\begin{array}{c}1.6 \pm 0 \\
-\end{array}$ \\
\hline (46) Solidity & $\begin{array}{c}0.9 \pm 0 \\
-\end{array}$ & $\begin{array}{c}1 \pm 0 \\
-\end{array}$ & $\begin{array}{c}1 \pm 0 \\
-\end{array}$ & $\begin{array}{c}1 \pm 0 \\
-\end{array}$ & $\begin{array}{c}1 \pm 0 \\
-\end{array}$ \\
\hline (47) Convexity & $\begin{array}{c}1 \pm 0 \\
-\end{array}$ & $\begin{array}{c}1 \pm 0 \\
-\end{array}$ & $\begin{array}{c}1 \pm 0 \\
-\end{array}$ & $\begin{array}{c}1 \pm 0 \\
-\end{array}$ & $1 \pm 0$ \\
\hline (48) Roundness & $\begin{array}{c}1.4 \pm 0 \\
-\end{array}$ & $\begin{array}{c}1.3 \pm 0 \\
-\end{array}$ & $\begin{array}{c}1.3 \pm 0 \\
-\end{array}$ & $\begin{array}{c}1.3 \pm 0 \\
-\end{array}$ & $\begin{array}{c}1.3 \pm 0 \\
-\end{array}$ \\
\hline (49) Compactness & $\begin{array}{c}1.2 \pm 0 \\
-\end{array}$ & $\begin{array}{c}1.1 \pm 0 \\
-\end{array}$ & $\begin{array}{c}1.2 \pm 0 \\
-\end{array}$ & $\begin{array}{c}1.2 \pm 0 \\
-\end{array}$ & $\begin{array}{c}1.1 \pm 0 \\
-\end{array}$ \\
\hline
\end{tabular}

$n$, Number of cells; < indicates significantly smaller with $P \leq 0.05 ;<<$ indicates significantly smaller with $\mathrm{p} \leq 0.01 ;<<<$ indicates significantly smaller with $\mathrm{P} \leq 0.001$; one-way ANOVA, followed by a Newman-Keuls test. Values significantly different in one cluster $v s$. all others were highlighted in gray. 
Diffusion of the neuronal tracer (biocytin) contained in the patch pipette during electrophysiological recordings allowed, after the fixation of slices and diaminobenzidine $(D A B)$-based processing to visualize the recorded neurons. Dendritic morphologies of the previously physiologically characterized neurons were successfully revealed for 110 out of the 289 recorded neurons. Their morphological variables were extracted from 3D Neurolucida reconstructions (Table 8, Fig. 3A).

A

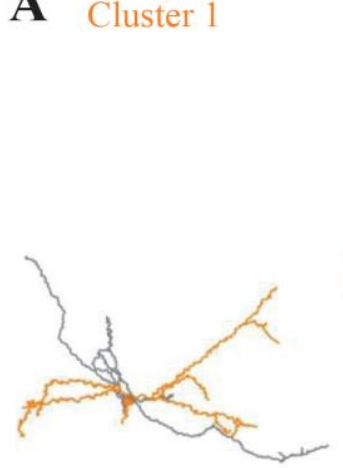

Cluster $2 \mathrm{a}$

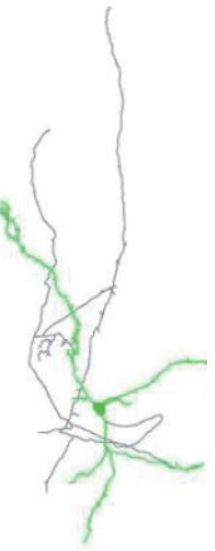

B

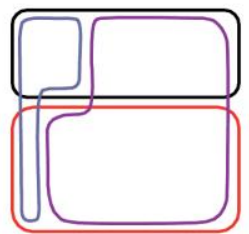

Cluster 2b
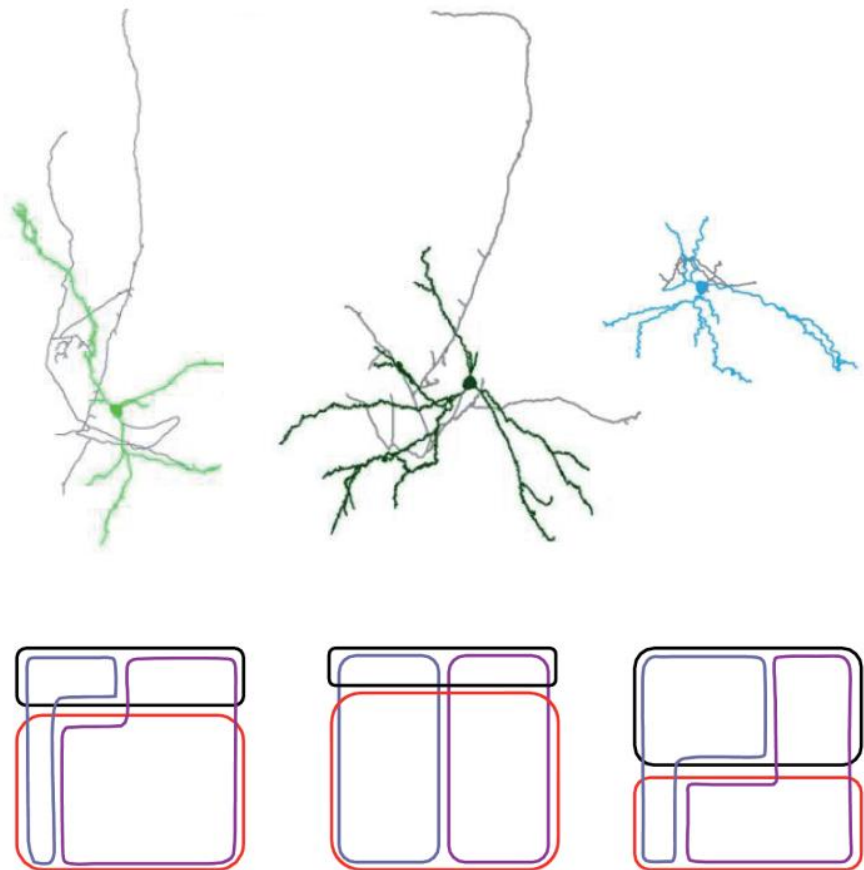

口NA (-)

$\square$ NA (+) $\quad \square$ 5-HT (-) $\square$ 5-HT (+)
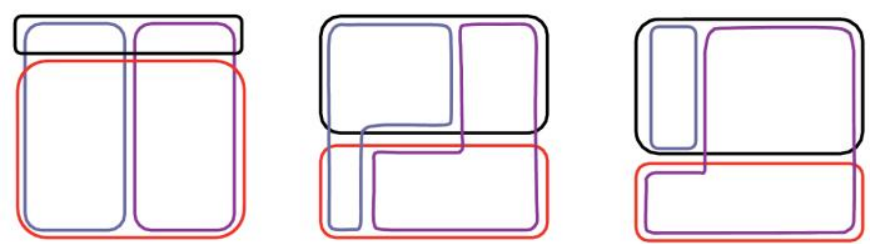

Cluster $3 b$

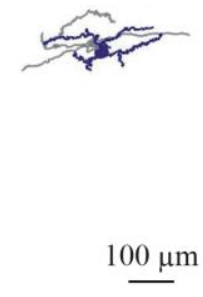

$\underline{00 \mu \mathrm{m}}$

Fig. 3. Morphological and pharmacological features of VLPO neurons. A, Neurolucida reconstructions of typical neurons from each cluster. Axons are represented in gray and dendrites are color coded according to their cluster. The pial surface is downward. B, Venn diagram of neuronal responses to NA $(n=189)$ and 5-HT $(n=130)$ applications.

Table 8. Dendritic properties among VLPO neurons

\begin{tabular}{lccccc}
\hline & $\begin{array}{c}\text { Cluster 1 } \\
(n=24)\end{array}$ & $\begin{array}{c}\text { Cluster 2a } \\
(n=26)\end{array}$ & $\begin{array}{c}\text { Cluster 2b } \\
(n=11)\end{array}$ & $\begin{array}{c}\text { Cluster 3a } \\
(n=20)\end{array}$ & $\begin{array}{c}\text { Cluster 3b } \\
(n=29)\end{array}$ \\
\hline (50) Nb of dendrites & $3.5 \pm 0.2$ & $3.7 \pm 0.2$ & $4.0 \pm 0.5$ & $3.9 \pm 0.2$ & $3.6 \pm 0.2$ \\
\hline
\end{tabular}




\begin{tabular}{|c|c|c|c|c|c|}
\hline $\begin{array}{l}\text { (51) Total length } \\
(\mu \mathrm{m})\end{array}$ & $\begin{array}{c}- \\
2092.3 \pm 242.0 \\
-\end{array}$ & $\begin{array}{c}- \\
1923.7 \pm 190.3 \\
-\end{array}$ & $\begin{array}{c}- \\
2504.5 \pm 391.0 \\
2 \mathrm{~b}>3 \mathrm{~b}\end{array}$ & $\begin{array}{c}- \\
2126.8 \pm 244.4 \\
-\end{array}$ & $\begin{array}{c}- \\
1440.2 \pm 151.7 \\
3 b<2 b\end{array}$ \\
\hline (52) Surface $\left(\mu \mathrm{m}^{2}\right)$ & $\begin{array}{c}5004.2 \pm 781.9 \\
-\end{array}$ & $\begin{array}{c}4648.1 \pm 627.0 \\
-\end{array}$ & $\begin{array}{c}6931.4 \pm 1158 \\
2 b>3 b\end{array}$ & $\begin{array}{c}5053.5 \pm 677.5 \\
-\end{array}$ & $\begin{array}{c}3690.8 \pm 330.5 \\
3 b<2 b\end{array}$ \\
\hline (53) Volume $\left(\mu \mathrm{m}^{3}\right)$ & $\begin{array}{c}1519.7 \pm 279.6 \\
1<2 \mathrm{~b}\end{array}$ & $\begin{array}{c}1359.7 \pm 219.4 \\
2 \mathrm{a}>2 \mathrm{~b}\end{array}$ & $\begin{array}{c}2277.8 \pm 460.0 \\
2 b>1,2 a, 3 a>>3 b\end{array}$ & $\begin{array}{c}1426.1 \pm 224.6 \\
3 a<2 b\end{array}$ & $\begin{array}{c}1149.0 \pm 122.6 \\
3 b<<2 b\end{array}$ \\
\hline $\begin{array}{l}\text { (54) Length/surface } \\
\left(\mu \mathrm{m}^{-1}\right)\end{array}$ & $\begin{array}{c}0.6 \pm 0.1 \\
1>3 b\end{array}$ & $\begin{array}{c}0.5 \pm 0.1 \\
-\end{array}$ & $\begin{array}{c}0.4 \pm 0.0 \\
-\end{array}$ & $\begin{array}{c}0.5 \pm 0.1 \\
3 a>3 b\end{array}$ & $\begin{array}{c}0.4 \pm 0.0 \\
3 \mathrm{~b}<1,3 \mathrm{a}\end{array}$ \\
\hline $\begin{array}{l}\text { (55) Highest order } \\
\text { segment }\end{array}$ & $\begin{aligned} & 5.9 \pm 0.5 \\
&-\end{aligned}$ & $\begin{array}{c}5.6 \pm 0.5 \\
-\end{array}$ & $\begin{array}{c}5.8 \pm 0.5 \\
-\end{array}$ & $\begin{array}{c}6.2 \pm 0.8 \\
-\end{array}$ & $\begin{array}{c}4.8 \pm 0.4 \\
-\end{array}$ \\
\hline (56) Tortuosity & $\begin{array}{c}1.3 \pm 0.0 \\
-\end{array}$ & $\begin{array}{c}1.3 \pm 0.0 \\
-\end{array}$ & $1.4 \pm 0.0$ & $1.3 \pm 0.0$ & $\begin{array}{c}1.4 \pm 0.0 \\
-\end{array}$ \\
\hline (57) Nodes & $12.8 \pm 1.8$ & $11.8 \pm 1.6$ & $13.0 \pm 1.7$ & $14.3 \pm 2.3$ & $\begin{array}{c}9.3 \pm 1.2 \\
-\end{array}$ \\
\hline (58) Sholl (100) & $\begin{array}{c}687.6 \pm 52.0 \\
-\end{array}$ & $\begin{array}{c}646.5 \pm 34.9 \\
-\end{array}$ & $\begin{array}{c}968.4 \pm \underset{-}{149.6} \\
-\end{array}$ & $\begin{array}{c}740.4 \pm \underset{-}{7} \\
72.16\end{array}$ & $\begin{array}{c}637.3 \pm 54.3 \\
-\end{array}$ \\
\hline (59) Sholl (200) & $\begin{array}{c}639.6 \pm 94.8 \\
-\end{array}$ & $\begin{array}{c}558.7 \pm 57.6 \\
-\end{array}$ & $\begin{array}{c}832.5 \pm 132.3 \\
-\end{array}$ & $\begin{aligned} & 681.0 \pm 109.6 \\
&-\end{aligned}$ & $\begin{array}{c}489.2 \pm 74.1 \\
-\end{array}$ \\
\hline (60) Sholl (300) & $\begin{array}{c}439.8 \pm 87.3 \\
1>3 b\end{array}$ & $\begin{array}{c}365.4 \pm 64.4 \\
-\end{array}$ & $\begin{array}{c}390.4 \pm 115.3 \\
-\end{array}$ & $\begin{array}{c}377.2 \pm 61.4 \\
-\end{array}$ & $\begin{array}{c}181.1 \pm 36.4 \\
3 b<<1\end{array}$ \\
\hline (61) Sholl (400) & $\begin{array}{c}325.2 \pm 78.1 \\
-\end{array}$ & $\begin{array}{c}353.11 \pm 85.3 \\
-\end{array}$ & $\begin{array}{c}313.3 \pm 123.1 \\
-\end{array}$ & $\begin{array}{c}328.2 \pm 103.0 \\
-\end{array}$ & $132.2 \pm 41.5$ \\
\hline (62) Spine 1 & $\begin{array}{c}3.2 \pm \\
\pm \\
-\end{array}$ & $\begin{array}{c}14.3 \pm 3.8 \\
-\end{array}$ & $2.6 \pm 0.9$ & $\begin{array}{c}5.0 \pm 1.4 \\
-\end{array}$ & $11.0 \pm 3.7$ \\
\hline (63) Spine 2 & $\begin{array}{c}29.6 \pm 5.5 \\
-\end{array}$ & $25.9 \pm 4.4$ & $29.5 \pm 20.1$ & $22.5 \pm 6.1$ & $27.2 \pm 6.2$ \\
\hline (64) Spine 3 & $\begin{array}{c}39.3 \pm 8.8 \\
-\end{array}$ & $\begin{array}{c}26.6 \pm 7.8 \\
-\end{array}$ & $\begin{array}{c}52.1 \pm 35.3 \\
-\end{array}$ & $\begin{array}{c}42.1 \pm 10.0 \\
-\end{array}$ & $16.1 \pm 3.4$ \\
\hline (65) Spine 4 & $\begin{array}{c}23.6 \pm 7.2 \\
1<2 b\end{array}$ & $\begin{array}{c}12.2 \pm 4.1 \\
2 \mathrm{a}<2 \mathrm{~b}\end{array}$ & $\begin{array}{c}47.3 \pm 33.4 \\
2 \mathrm{~b}>1,2 \mathrm{a}, 3 \mathrm{~b}\end{array}$ & $\begin{array}{c}25.5 \pm 9.9 \\
-\end{array}$ & $\begin{array}{c}11.1 \pm 3.5 \\
3 b<2 b\end{array}$ \\
\hline (66) Spine 5 & $\begin{array}{c}6.6 \pm 2.2 \\
-\end{array}$ & $\begin{array}{c}5.7 \pm 2.2 \\
-\end{array}$ & $8.6 \pm 5.1$ & $\begin{array}{c}8.1 \pm 2.9 \\
-\end{array}$ & $\begin{array}{c}5.0 \pm 2.4 \\
-\end{array}$ \\
\hline (67) Spine 6 & $\begin{array}{c}4.2 \pm 1.6 \\
-\end{array}$ & $\begin{array}{c}6.3 \pm 3.1 \\
-\end{array}$ & $\begin{array}{c}3.1 \pm 2.7 \\
-\end{array}$ & $\begin{array}{c}7.6 \pm 4.9 \\
-\end{array}$ & $\begin{array}{c}4 \pm 1.9 \\
-\end{array}$ \\
\hline (68) Spine density & $\begin{array}{c}1.5 \pm 0.3 \\
-\end{array}$ & $\begin{array}{c}1.4 \pm 0.3 \\
- \\
\end{array}$ & $\begin{array}{c}1.5 \pm 0.9 \\
- \\
\end{array}$ & $\begin{array}{c}1.9 \pm 0.6 \\
-\end{array}$ & $\begin{array}{c}1.2 \pm 0.3 \\
- \\
\end{array}$ \\
\hline
\end{tabular}

$n$, Number of cells; < indicates significantly smaller with $P \leq 0.05 ;<<$ indicates significantly smaller with $P \leq 0.01 ;<<<$ indicates significantly smaller with $P \leq 0.001$; one-way ANOVA, followed by a Newman-Keuls test. Values significantly different in one cluster $v s$. all others were highlighted in gray.

We examined and compared the dendritic properties of VLPO neurons among the 5 neuronal clusters (Table 8). In particular, we found that neurons from cluster $2 \mathrm{~b}$ are distinguished from other neurons by their bigger dendritic volume (53). Although not statistically different from all other clusters, neurons from cluster $3 b$ were also found to be smaller (51-53), with in particular a shorter dendritic sholl at $300 \mu \mathrm{m}$ (60; Table 8).

Axonal properties of VLPO neurons were also extracted from Neurolucida reconstructions when the total axonal length of the reconstructed neuron exceeded $1 \mathrm{~mm}$ (Table 9). In some 
cases, axons were cut at the surface of the slices and were not possible to track (69, Table 9). None of these axonal parameters are distinguish specifically in a cluster compared to others (Table 9). Nevertheless, we established that in $43 \%$ of the cases the axon arose directly from the soma, whereas in $37 \%$ of the reconstructed neurons the axon arose from a primary dendrite and in remaining $20 \%$ of the cases, the axon arose from the secondary or even from the tertiary dendrite. The mean distance of the axonal arising was at $12.94 \pm 2.21 \mu \mathrm{m}$ from the soma.

Table 9. Axonal properties among VLPO neurons

\begin{tabular}{|c|c|c|c|c|c|}
\hline & $\begin{array}{c}\text { Cluster } 1 \\
(n=9)\end{array}$ & $\begin{array}{c}\text { Cluster 2a } \\
(n=10) \\
\end{array}$ & $\begin{array}{c}\text { Cluster 2b } \\
(n=5) \\
\end{array}$ & $\begin{array}{c}\begin{array}{c}\text { Cluster 3a } \\
(n=7)\end{array} \\
\end{array}$ & $\begin{array}{c}\text { Cluster 3b } \\
(n=10)\end{array}$ \\
\hline \multirow[t]{2}{*}{ (69) Axon > $1000 \mu \mathrm{m}(\%$} & $\%$ & 62.5 & 55.6 & 41.2 & 43.5 \\
\hline & - & - & - & - & - \\
\hline (70) Length $(\mu \mathrm{m})$ & $\begin{array}{c}3377.9 \pm 383.9 \\
-\end{array}$ & $\begin{array}{c}2665.7 \pm 314.4 \\
-\end{array}$ & $\begin{array}{c}3336.1 \pm 865.4 \\
-\end{array}$ & $\begin{array}{c}2808.1 \pm 452.4 \\
-\end{array}$ & $\begin{array}{c}2438.0 \pm 290.4 \\
-\end{array}$ \\
\hline (71) Surface $\left(\mu \mathrm{m}^{2}\right)$ & $\begin{array}{c}3750.9 \pm 760.4 \\
-\end{array}$ & $2142.6 \pm 436.5$ & $\begin{array}{c}3094.7 \pm 1015 \\
-\end{array}$ & $\begin{array}{c}2184.3 \pm 335.2 \\
-\end{array}$ & $\begin{array}{c}2342.4 \pm 459.6 \\
-\end{array}$ \\
\hline (72) Volume $\left(\mu \mathrm{m}^{3}\right)$ & $\begin{array}{c}550.6 \pm 190.7 \\
1>2 \mathrm{a}\end{array}$ & $\begin{array}{c}199.9 \pm 47.2 \\
2 \mathrm{a}<1\end{array}$ & $\begin{array}{c}385.1 \pm 70.8 \\
-\end{array}$ & $\begin{array}{c}180.6 \pm 26.4 \\
-\end{array}$ & $\begin{array}{c}253.0 \pm 53.6 \\
-\end{array}$ \\
\hline $\begin{array}{l}\text { (73) Length/surface } \\
\left(\mu \mathrm{m}^{-1}\right)\end{array}$ & $\begin{array}{c}1.0 \pm 0.1 \\
-\end{array}$ & $1.3 \pm 0.1$ & $\begin{array}{c}1.0 \pm 0.1 \\
-\end{array}$ & $\begin{array}{c}1.3 \pm 0.1 \\
-\end{array}$ & $\begin{array}{c}1.1 \pm 0.1 \\
-\end{array}$ \\
\hline (74) Tortuosity & $0.6 \pm 0.1$ & $0.5 \pm 0.1$ & $0.6 \pm 0.4$ & $0.4 \pm 0.0$ & $0.6 \pm 0.2$ \\
\hline (75) Nodes & $\begin{array}{c}35.2 \pm 19.7 \\
-\end{array}$ & $\begin{array}{c}26.8 \pm 7.7 \\
-\end{array}$ & $\begin{array}{c}34.2 \pm 25.0 \\
-\end{array}$ & $14.5 \pm 2.8$ & $\begin{array}{c}15.7 \pm 4.8 \\
-\end{array}$ \\
\hline (76) Sholl (100) & $\begin{array}{c}520.8 \pm 93.7 \\
-\end{array}$ & $\begin{aligned} & 596.3 \pm 109.8 \\
&-\end{aligned}$ & $\begin{aligned} & 379.6 \pm 117.7 \\
&-\end{aligned}$ & $\begin{array}{c}294.7 \pm 76.4 \\
-\end{array}$ & $\begin{aligned} & 483.4 \pm 130.4 \\
&-\end{aligned}$ \\
\hline (77) Sholl (200) & $\begin{aligned} 792.7 & \pm 149.9 \\
& -\end{aligned}$ & $\begin{aligned} 706.7 & \pm 107.8 \\
& -\end{aligned}$ & $\begin{aligned} 796.1 & \pm 273.3 \\
& -\end{aligned}$ & $\begin{array}{c}431.5 \pm 61.8 \\
-\end{array}$ & $\begin{aligned} & 875.6 \pm 250.3 \\
&-\end{aligned}$ \\
\hline (78) Sholl (300) & $677.8 \pm 189.0$ & $\begin{array}{c}418.4 \pm 90.8 \\
-\end{array}$ & $\begin{aligned} & 447.1 \pm 197.8 \\
&-\end{aligned}$ & $414.5 \pm 36.1$ & $\begin{array}{c}405.8 \pm 118.7 \\
-\end{array}$ \\
\hline (79) Sholl (400) & $\begin{array}{c}1386.5 \pm 465.8 \\
- \\
\end{array}$ & $\begin{aligned} & 944.2 \pm 423.7 \\
&- \\
&\end{aligned}$ & $\begin{array}{c}1713.4 \pm 903.9 \\
- \\
\end{array}$ & $\begin{array}{c}1671.4 \pm 467.7 \\
- \\
\end{array}$ & $\begin{aligned} & 673.3 \pm 262.8 \\
&- \\
&\end{aligned}$ \\
\hline
\end{tabular}

$n$, Number of cells; $<$ indicates significantly smaller with $P \leq 0.05 ;<<$ indicates significantly smaller with $P \leq 0.01 ;<<<$ indicates significantly smaller with $P \leq 0.001$; one-way ANOVA, followed by a Newman-Keuls test. Values significantly different in one cluster $v s$. all others were highlighted in gray.

\section{Spatial mapping of the distinct neuronal subpopulations into the VLPO}

Reconstructed neurons were spatially mapped across three different brain sections of the VLPO, rostral to caudal (Fig. 4). The superimposed mapping of the neuronal subpopulations within the VLPO did not reveal a preferred location of any subpopulations that seem homogeneously spread. 

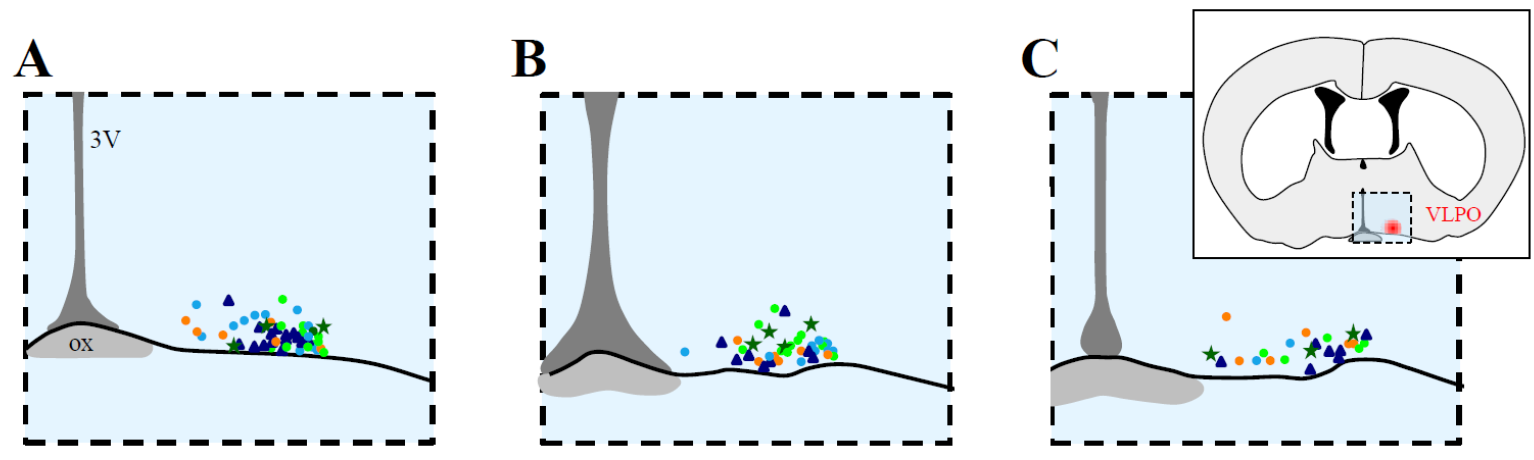

- Cluster $1 \quad$ Cluster 2a $\quad \star$ Cluster 2b Cluster 3a

$\Delta$ Cluster $3 b$

Fig. 4. Schematic distribution of the biocytin-labeled neurons within the VLPO. A, B and $\mathbf{C}$ represent three different planes at $\sim 0.2 \mathrm{~mm}$ intervals rostral to caudal, around Bregma $0 \mathrm{~mm}$, where VLPO neurons were recorded. The five subpopulations of VLPO neurons are color coded. In the plane (A) are represented 6 neurons from cluster 1 (yellow, circle); 10 neurons from cluster 2a (light green, circle), 4 neurons from cluster 2b (dark green, star), 12 neurons from cluster $3 \mathrm{a}$ (light blue, circle) and 15 neurons from cluster $3 \mathrm{~b}$ (dark blue, triangle). In the plane (B) are represented 6 neurons from cluster 1 (yellow); 11 neurons from cluster 2a (light green), 4 neurons from cluster $2 \mathrm{~b}$ (dark green), 8 neurons from cluster $3 \mathrm{a}$ (light blue) and 7 neurons from cluster $3 b$ (dark blue). In the plane (C), are represented 6 neurons from cluster 1 (yellow); 5 neurons from cluster 2a (light green), 3 neurons from cluster $2 \mathrm{~b}$ (dark green), 2 neurons from cluster $3 \mathrm{a}$ (light blue) and 7 neurons from cluster $3 \mathrm{~b}$ (dark blue). Insert: Coronal brain section showing the localization of the zoom presented in (A-C). Abbreviations: $3 \mathrm{~V}$, third ventricle; ox, optic chiasm.

\section{Pharmacological properties of VLPO neurons}

In the VLPO, sleep-promoting neurons are usually identified according to their inhibitory response to bath application of NA (Gallopin et al. 2000; Gallopin et al. 2005; Moore et al. 2012; Liu et al. 2013; Varin et al. 2015). Moreover, their response to 5-HT was also reported to segregate two subsets of VLPO sleep-promoting neurons (Gallopin et al. 2005). Therefore 189 and 132 neurons out of the 289 neurons of our data set were respectively tested for their response to NA (50 $\mu \mathrm{M}$ for $30 \mathrm{~s})$ and to $5-\mathrm{HT}(100 \mu \mathrm{M}$ for $5 \mathrm{~s})$ application. 111 of these neurons were tested for both neurotransmitters. 
We found that on average $42 \%$ of VLPO neurons were hyperpolarized by NA application. The highest proportion of NA (-) neurons was observed in the cluster $3(61 \% ; n=51)$, whereas neurons in cluster 1 and 2 were mainly NA (+) $(60 \%, n=18$ and 76\%, $n=55$ respectively, Fig. 3B). To determine if NA (-) vs. NA (+) neurons displayed specific features, we compared their properties. Thus, we found in cell-attached recordings that NA (-) cells spontaneously discharged at higher firing rate than NA (+) cells $(4.7 \pm 0.7 \mathrm{~Hz} v s .2 .8 \pm 0.3 \mathrm{~Hz}$ respectively; $P$ $<0.01$; Mann-Whitney $U$-test) and were more likely endowed with an LTS (68\% vs. $44 \%$ of NA (+) cells; $P<0.001)$. Only few others electrophysiological differences were observed comparing NA (+) and NA (-) neurons. In particular, NA (-) cells had a significantly higher input resistance, membrane time constant and $\mathrm{G}_{\text {hyp }}(791.4 \pm 45.9$ vs. $648.1 \pm 44.4 \mathrm{M} \Omega ; P<$ $0.010 ; 39.2 \pm 2.3$ vs. $33.4 \pm 2.1 \mathrm{~ms} ; P<0.019$ and $573.3 \pm 33.8 v s .437 .9 \pm 23.8 \mathrm{M} \Omega ; P<0.002$ Mann-Whitney $U$-test); whereas NA (+) cells had a significantly higher $\Delta \mathrm{G}_{\text {sag }}$ and first spike delay $(78.3 \pm 1.6 v s .71 .5 \pm 1.9 \% ; P<0.005 ; 141.7 \pm 12.7 v s .99 .5 \pm 8.6 \mathrm{~ms} ; P<0.005$, MannWhitney $U$-test).

Morphologically, NA (+) neurons were shown to have bigger somata than NA (-) neurons. Their somatic perimeter was of $61.1 \pm 1.2 v s .55 .9 \pm 1.4 \mu \mathrm{m}$ respectively $(P<0.039$, MannWhitney $U$-test) with a higher mean dendritic length of $5922.3 \pm 286.8 v s .4425 .5 \pm 310.1 \mu \mathrm{m}$ $(P<0.011$, Mann-Whitney $U$-test). Here again, no correlation was found between the somatic shape and the neuronal response to NA. We found that only $41 \%$ of the triangular/multipolar cells in the VLPO were inhibited by NA, and conversely that $62 \%$ fusiform/bipolar cells excited by NA, confirming the previous observation than on the opposite to rat observations, somata of mice VLPO neurons are not predictive of their response to NA (Wang et al. 2012).

In the 130 neurons tested for their response to 5-HT application, $72 \%$ were depolarized by the 5-HT. Cluster $2 \mathrm{~b}$ presented the smallest proportion of 5-HT $(+)$ neurons $(50 \% ; n=16)$, close to the amount of 5-HT (+) neurons observed in cluster 3a (55\%). Neurons from cluster 1, 2a 
and $3 \mathrm{~b}$ included higher proportion of 5-HT (+) neurons, 84.2, 75 and $82.1 \%$ respectively. Interestingly, the comparison of 5-HT (+) and 5-HT (-) neuronal features revealed the existence of only one discriminative parameter: the capacitance $(58.3 \pm 2.9 v s .74 .5 \pm 6.9 \mathrm{pF}$ respectively; $P<0.04$, Mann-Whitney $U$-test).

In the 110 neurons both tested for their response to NA and 5-HT, we found 44\% of NA (+)/5HT (+) cells, $32 \%$ of NA (-)/5-HT (+) cells, $20 \%$ of NA (-)/5-HT (-) cells, and $12 \%$ of NA (+)/5-HT (-) cells. These four populations had similar electrophysiological and morphological properties, except for the NA (+)/5-HT (-) neurons that were characterized by a low input resistance of $491.4 \pm 107.4 \mathrm{M} \Omega$, high membrane capacitance of $100.0 \pm 13.2 \mathrm{pF}$ and $\Delta \mathrm{G}_{\text {sag }}$ of $84.8 \pm 3.3 \%$ and a long first spike delay of $220.8 \pm 62.2 \mathrm{~ms}$ compared to the other groups.

To determine whether the inclusion of these pharmacological responses could provide discriminative parameter to define VLPO subclasses, we performed a Ward's clustering on these 111 neurons tested for both the NA and 5-HT effects. However, this approach provided a dendrogram with a smaller distance of aggregation and with a lower silhouette value. Therefore, these pharmacological properties were only kept as a read out in each identified clusters (Fig. 3B).

\section{Spontaneous synaptic postsynaptic currents}


Spontaneous excitatory and inhibitory postsynaptic currents (sEPSCs and sIPSCs) were recorded in 66 neurons out of the 289 cells of our database. However, due to a high intercellular variability, no significant differences were observed between the clusters, neither regarding the amplitude nor the frequency of both sEPSCs (from $18.97 \pm 2.56$ to $21.88 \pm 1.33 \mathrm{pA} ; P>0.611$; and $0.91 \pm 0.14$ to $1.67 \pm 0.44 \mathrm{~Hz} ; P>0.829$, one-way ANOVA test) and sIPSCs (from 13.91 \pm 2.47 to $15.46 \pm 0.68 \mathrm{pA} ; P>0.810 ;$ and $0.14 \pm 0.1$ to $0.79 \pm 0.43 \mathrm{~Hz} ; P>0.294$, one-way ANOVA; Fig. 6).

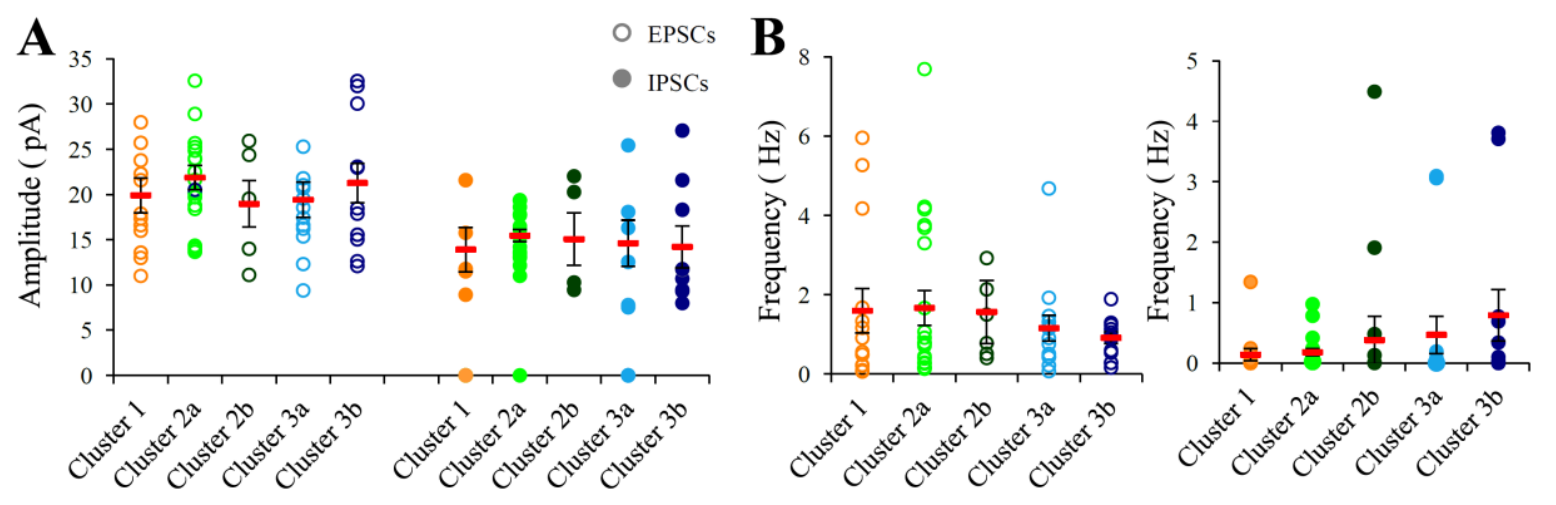

Fig. 6. Recording of spontaneous excitatory and inhibitory synaptic inputs to VLPO neurons. A, The amplitude of both sEPSCs and sIPSCs revealed no statistical differences among the 5 clusters. B, The frequency of both sEPSCs (left panel) and sIPSCs (right panel) revealed no statistical differences among the 5 clusters. 


\section{Discussion}

Given the critical role of the VLPO in sleep induction and maintenance, the characterization of its neuronal populations is essential for a better understanding of their role in sleep regulation. Using unsupervised clustering method on recorded neurons from acute slices of mice, we have defined for the first time the existence of five distinct cell subpopulations in the VLPO.

In this study, we recorded 289 neurons from the VLPO. 39 electrophysiological parameters were measured for each neuron. To define the existence of distinct neuronal subpopulations among VLPO neurons, we used the Ward's method which is usually used to group cells with large similarities and to avoid a personal bias of interpretation (Cauli et al. 1997; Karagiannis et al. 2009). Here, we performed a Ward's clustering on 18 electrophysiological parameters. Interestingly, we found that intrinsic burst firing measured in cell-attached configuration was a strong discriminative feature. 19\% of VLPO neurons were shown to display intrinsic burst. This result is in good agreement with a previous observation reporting $14 \%$ of burst firing neurons in the VLPO (Morairty et al. 2004). These neurons segregated mainly in the cluster 1 (83\%) and to a lesser extent in the cluster $3(17 \%)$. Neurons from cluster 1 were also characterized by their short lasting and low amplitude AHP compared to neurons from the other clusters. Moreover, their second action potential evoked by a current pulse was significantly smaller. Neurons from cluster 1 frequently displayed an LTS (80\%) and 40\% of these neurons were inhibited by NA application. These two hallmarks of sleep-promoting neurons suggest that at least some neurons from the cluster 1 could correspond to sleep-promoting neurons.

Cluster 2 VLPO neurons were composed of two distinct subsets: cluster 2a and cluster 2b cells, both exhibiting a high proportion of NA (+) cells (74 and $81.9 \%$ respectively) with a low occurrence of LTS (4.7 and $21.1 \%$ respectively). In particular, neurons from the cluster $2 \mathrm{~b}$ 
strongly differentiated themselves from all other clusters. Pharmacologically, they were shown to contain the highest amount of 5-HT (-) neurons (50\%). Electrophysiologically, Cluster $2 \mathrm{~b}$ cells were characterized by a significantly higher membrane capacitance, a long delay to spiking and a strong outward rectification in the positive direction. Interestingly, these features were already reported to be the main characteristics distinguishing cholinergic basal forebrain neurons (Khateb et al. 1993; Unal et al. 2012). These results suggest that on the opposite of rat where cholinergic neurons are confined into the basal forebrain, these neurons could be more sparsely segregated in mice brains. We speculate that neurons from cluster 2 a could therefore correspond to local interneurons, whereas neurons from cluster $2 \mathrm{~b}$ could correspond to cholinergic neurons.

Neurons from cluster 3 frequently exhibited an LTS (84\%) and were in majority inhibited by NA application (61\%). Interestingly, these neurons segregated into two different subpopulations, cluster $3 \mathrm{a}$ and $3 \mathrm{~b}$. Neurons from cluster $3 \mathrm{~b}$ in particular were shown to have the smallest membrane resistance, the largest voltage sags, the lowest rheobase, the longer rebound duration, and numerous action potentials generated by their post-inhibitory rebound, compared to other VLPO neurons. In general, dendritic arborization of VLPO neurons were simple and quite similar among the clusters. Hence, neurons from cluster $3 \mathrm{~b}$ displayed a particularly small somato dendritic tree. Finally, cluster $3 \mathrm{~b}$ neurons also contained the highest proportion of 5-HT (+) neurons $(82 \%)$.

To examine if synaptic inputs could differentiate VLPO neurons, we recorded sEPSCs and sIPSCS of 66 cells. However, we observed an important variation of sEPSCs and sIPSCS amplitude and frequency within each cluster. Thus, no statistical differences were found among VLPO clusters. 
The specific features of VLPO neuronal clusters that we have defined in this study suggest that these different subpopulations could be involved in distinct aspects of sleep physiology. The role of some VLPO neurons in the thermoregulation being previously reported (Lu et al. 2000), we speculate that one of these clusters could be involved in this function. Future studies will be needed to investigate the physiological roles of these 5 neuronal subpopulations.

\section{Conclusions}

In the present study, we identified 5 distinct neuronal subpopulations in the VLPO, characterized by specific electrophysiological, morphological and pharmacological properties that might be involved in specific functions of sleep regulation. These different subpopulations suggest a complex neuronal network organization within the VLPO.

\section{Acknowledgments}

This work was supported by a grant from ANR-12-JSV4-001-01, the Centre National de la Recherche Scientifique (CNRS), the French Institute of Health and Medical Research (Inserm) and ESPCI ParisTech. We are grateful to Judith Pineau and Aude Duhamel for technical support. The authors declare no competing financial interests. 


\section{References}

Ascoli GA, Onso-Nanclares L, Anderson SA, et al (2008) Petilla terminology: nomenclature of features of GABAergic interneurons of the cerebral cortex. Nat Rev Neurosci 9:557-568.

Cauli B, Audinat E, Lambolez B, et al (1997) Molecular and physiological diversity of cortical nonpyramidal cells. J Neurosci 17:3894-3906.

Fort P, Bassetti CL, Luppi PH (2009) Alternating vigilance states: New insights regarding neuronal networks and mechanisms. Eur J Neurosci 29:1741-1753.

Gallopin T, Fort P, Eggermann E, et al (2000) Identication of sleep-promoting neurons in vitro. Nature 404:3-6.

Gallopin T, Luppi PH, Cauli B, et al (2005) The endogenous somnogen adenosine excites a subset of sleep-promoting neurons via A2A receptors in the ventrolateral preoptic nucleus. Neuroscience 134:1377-1390.

Gallopin T, Luppi PH, Rambert FA, et al (2004) Effect of the wake-promoting agent modafinil on sleep-promoting neurons from the ventrolateral preoptic nucleus: an in vitro pharmacologic study. Sleep 27:19-25.

Karagiannis A, Gallopin T, David C, et al (2009) Classification of NPY-expressing neocortical interneurons. J Neurosci 29:3642-3659.

Khateb A, Fort P, Alonso A, et al (1993) Pharmacological and immunohistochemical evidence for serotonergic modulation of cholinergic nucleus basalis neurons. Eur J Neurosci 5:541547.

Kim U, McCormick DA (1998) The functional influence of burst and tonic firing mode on synaptic interactions in the thalamus. J Neurosci 18:9500-16.

Liu Y-W, Li J, Ye J-H (2010) Histamine regulates activities of neurons in the ventrolateral preoptic nucleus. J Physiol 588:4103-16.

Liu Y-W, Zuo W, Ye J-H (2013) Propofol stimulates noradrenalin-inhibited neurons in the ventrolateral preoptic nucleus by reducing GABAergic inhibition. Anesth Analg 117:35863.

Lu J, Greco MA, Shiromani P, Saper CB (2000) Effect of lesions of the ventrolateral preoptic 
nucleus on NREM and REM sleep. J Neurosci 20:3830-3842.

Matsuo S-I, Jang I-S, Nabekura J, Akaike N (2003) alpha 2-Adrenoceptor-mediated presynaptic modulation of GABAergic transmission in mechanically dissociated rat ventrolateral preoptic neurons. J Neurophysiol 89:1640-1648.

McCarren HS, Chalifoux MR, Han B, et al (2014) 2-Adrenergic Stimulation of the Ventrolateral Preoptic Nucleus Destabilizes the Anesthetic State. J Neurosci 34:1638516396. doi: 10.1523/JNEUROSCI.1135-14.2014

Moore JT, Chen J, Han B, et al (2012) Direct activation of sleep-promoting VLPO neurons by volatile anesthetics contributes to anesthetic hypnosis. Curr Biol 22:2008-16.

Morairty S, Rainnie D, McCarley R, Greene R (2004) Disinhibition of ventrolateral preoptic area sleep-active neurons by adenosine: a new mechanism for sleep promotion. Neuroscience 123:451-457.

Perrenoud Q, Geoffroy H, Gauthier B, et al (2012) Characterization of Type I and Type II nNOS-Expressing Interneurons in the Barrel Cortex of Mouse. Front Neural Circuits 6:117.

Saint-Mleux B, Bayer L, Eggermann E, et al (2007) Suprachiasmatic modulation of noradrenaline release in the ventrolateral preoptic nucleus. J Neurosci 27:6412-6416.

Saint-Mleux B, Eggermann E, Bisetti A, et al (2004) Nicotinic enhancement of the noradrenergic inhibition of sleep-promoting neurons in the ventrolateral preoptic area. $\mathbf{J}$ Neurosci 24:63-67.

Saper CB, Fuller PM, Pedersen NP, et al (2010) Sleep state switching. Neuron 68:1023-1042.

Saper CB, Scammell TE, Lu J (2005) Hypothalamic regulation of sleep and circadian rhythms. Nature 437:1257-1263.

Scharbarg E, Daenens M, Lemaître F, et al (2016) Astrocyte-derived adenosine is central to the hypnogenic effect of glucose. Sci Rep 6:19107.

Sherin JE, Elmquist JK, Torrealba F, Saper CB (1998) Innervation of histaminergic tuberomammillary neurons by GABAergic and galaninergic neurons in the ventrolateral preoptic nucleus of the rat. J Neurosci 18:4705-4721.

Sherin JE, Shiromani PJ, McCarley RW, Saper CB (1996) Activation of ventrolateral preoptic neurons during sleep. Science 271:216-9. 
Sholl DA (1953) Dendritic organization in the neurons of the visual and motor cortices of the cat. J Anat 87:387-406.

Steriade M, Contreras D, Curró Dossi R, Nuñez A (1993) The slow ( $<1 \mathrm{~Hz}$ ) oscillation in reticular thalamic and thalamocortical neurons: scenario of sleep rhythm generation in interacting thalamic and neocortical networks. J Neurosci 13:3284-99.

Unal CT, Golowasch JP, Zaborszky L (2012) Adult mouse basal forebrain harbors two distinct cholinergic populations defined by their electrophysiology. Front Behav Neurosci 6:21.

Varin C, Rancillac A, Geoffroy H, et al (2015) Glucose Induces Slow-Wave Sleep by Exciting the Sleep-Promoting Neurons in the Ventrolateral Preoptic Nucleus: A New Link between Sleep and Metabolism. J Neurosci 35:9900-11.

Von Economo C (1930) Sleep as a problem of localization. J Nerv Ment Dis 249-259.

Wang Q, Yue X-F, Qu W-M, et al (2012) Morphine Inhibits Sleep-Promoting Neurons in the Ventrolateral Preoptic Area Via Mu Receptors and Induces Wakefulness in Rats. Neuropsychopharmacology 1-11.

Ward JH (1963) Hierarchical grouping to optimize an objective function. J. Am. Stat. Assoc. 58:236-244. 